\title{
Seasonal Variation of Antioxidant Capacity, Phenols, Minerals and Essential Oil Components of Sage, Spearmint and Sideritis Plants Grown at Different Altitudes
}

\author{
Antonios Chrysargyris ${ }^{1, *}$, Efstathios Evangelides ${ }^{2}$ and Nikolaos Tzortzakis ${ }^{1}$ (D) \\ 1 Department of Agricultural Sciences, Biotechnology and Food Science, Cyprus University of Technology, \\ Limassol 3603, Cyprus; nikolaos.tzortzakis@cut.ac.cy \\ 2 Department of Agriculture, Ministry of Agriculture, Rural Development and Environment, \\ Nicosia 1412, Cyprus; sevangelides@da.moa.gov.cy \\ * Correspondence: a.chrysargyris@cut.ac.cy
}

check for updates

Citation: Chrysargyris, A.; Evangelides, E.; Tzortzakis, N. Seasonal Variation of Antioxidant Capacity, Phenols, Minerals and Essential Oil Components of Sage, Spearmint and Sideritis Plants Grown at Different Altitudes. Agronomy 2021, 11, 1766. https://doi.org/10.3390/ agronomy11091766

Academic Editor: Erika Sabella

Received: 23 July 2021

Accepted: 31 August 2021

Published: 2 September 2021

Publisher's Note: MDPI stays neutral with regard to jurisdictional claims in published maps and institutional affiliations.

Copyright: (c) 2021 by the authors. Licensee MDPI, Basel, Switzerland. This article is an open access article distributed under the terms and conditions of the Creative Commons Attribution (CC BY) license (https:// creativecommons.org/licenses/by/ $4.0 /)$.

\begin{abstract}
Medicinal and aromatic plants are well appreciated for their antioxidant and biocidal activities, while great variation on these activities can be related to the species, environmental conditions and harvesting period. In the present study, the seasonal variation of the antioxidant activity, mineral content, yield and chemical composition of the essential oils (EOs) of sage (Salvia officinalis L.), sideritis (Sideritis perfoliata L. subsp. perfoliata) and spearmint (Mentha spicata L.) were tested under two different environmental conditions, each with a different altitude (namely mountainous and plain). Season affected total phenolic content and antioxidant capacity with increased values during winter and lower values during summer period. In summer, plants accumulated more Fe and had higher EO yield, while $\mathrm{P}$ and $\mathrm{Na}$ were accumulated more in winter. Altitude had a lesser effect on antioxidant capacity of the plants; however, increased minerals ( $\mathrm{N}, \mathrm{K}, \mathrm{Na}$ and $\mathrm{Ca}$ ) accumulation was found in plain areas. Sage plants had the highest antioxidant capacity, Zn content and EO yield. Sideritis had increased Fe content and spearmint plants revealed high N, Na and Mg levels. Furthermore, altitude and season had an impact on the content of main EOs components in all species. FRAP and ABTS were variably correlated with total phenols and minerals, depending on the species, season and altitude. In few cases, antioxidant activity was found to be inversely linked to some EO components (e.g., $\alpha$-thujone in sage). Finally, the antioxidant content, minerals and EO yield and composition of the examined MAPs were all altered by season and altitude. These findings can be utilized to implement sage, sideritis and spearmint farming in specific ecosystems, determining the season and areas for harvesting the plants, in order to produce high-value products.
\end{abstract}

Keywords: antioxidant status; ABTS; FRAP; altitude; total phenols; season; volatile compounds

\section{Introduction}

Medicinal and aromatic plants (MAPs), commonly known as herbs or spices, and their related plant extracts and essential oils (EOs) have been highly valued and widely used for centuries, of regardless the lack of scientific proof for their actual bioactive mechanisms and functions, which are still under research [1,2]. In the present day, dietary patterns recommend MAPs as functional foods, i.e., foods that provide physiological benefits in addition to the standard dietary requirements, preventing or postponing the onset of chronic diseases [3]. The global interest in MAPs is mirrored in the trade of raw material of MAPs, which is estimated to be around 440,000 tons per year, with a total value of 1.3 billion US dollars, 25\% of which is marketed in Europe [4].

Antioxidant-rich foods are popular because they can assist in reducing the burden of age-related chronic diseases by scavenging reactive oxygen species (ROS) [5]. As a result of their well-known antioxidant activity, MAPs have been the target of scientific research, food and pharmaceutical industries [1]. Plants produce a wide range of secondary metabolites, 
such as phenolic compounds, as part of their defense mechanism against oxidative damage caused by ROS and other abiotic and biotic stressors [2]. These compounds may also protect human health when MAPs and/or their components are consumed through diet [6]. Free radical scavenging, hydrogen atom donation, single oxygen quenching, metal ion chelation and activities as an oxidation substrate are all mechanisms involved in phenolic compounds' antioxidant activity [6].

More than 10,000 species of MAPs have been found in the Mediterranean region, as they are commonly used in the Mediterranean diet [7]. Although these species' major bioactive characteristics have been thoroughly described in ethnobotanical and ethnopharmacological studies [8], more research is needed to reveal and indicate their explicit medicinaland functional-related qualities as food supplements and unique antioxidants [9]. MAPs' biological activity and phytochemicals greatly vary according to their production area, climatic conditions and genetic material [10]. Specific environmental parameters such as the intensity of light and wind, the average temperature, UV-B radiation, ozone density, and partial carbon dioxide pressure may change between altitudes, according to Kofidis and Bosabalidis [11] and influence the quantitative composition of plant compound mixtures as volatiles and EOs [2]. Commonly, MAPs are harvested more than once per year, under favorable climatic conditions. Therefore, the basic knowledge about seasonal influences on the plant secondary metabolites is important to determine the optimal harvesting period, for high quality products $[12,13]$. Furthermore, the existence of secondary metabolites with antioxidant capacity, such as phenolic compounds, is frequently related with the bioactive properties of MAPs [14]. However, caution should be exercised before suggesting the use of MAPs in human diet, as large dosages of secondary metabolites and potentially dangerous compounds (e.g., heavy metals, anti-nutritional factors) have been shown to induce severe toxicity and significant health effects in some cases [15]. As a result, more research is required to assess potential toxicity and to determine the recommended daily allowance (RDA) limits, particularly for people with medical issues [16].

Apart from using MAPs as herbs and decoctions, their EOs have found applications in the food and pharmaceutical industries [17]. Several researches on EOs indicated substantial antioxidant [18,19] and antimicrobial properties [20,21], piquing interest in using EOs as natural antioxidants and antimicrobial agents rather than synthetic substances, as the latter are currently being chastised for their negative impacts on human health [20-22]. Despite the increased interest and large number of MAPs around the world, only about $10 \%$ among the EOs that are previously known have attracted interest because of their wide range of biological activities [1], and they are extensively used in the food, cosmetics and pharmaceutical industries today [23].

MAPs cultivation in Cyprus has excellent prospects because of the crops' low agrochemicals, irrigation water, manpower and energy requirements [24], as well as their resilience to harsh climatic conditions such as high temperatures, winds and drought [25]. All these important characteristics could contribute to the long-term development of rural communities while at the same time reducing the risks associated with MAP harvesting from the wild, reducing native populations [26]. Even though the soil and climatic conditions on the island are suitable for the growth of MAPs, their cultivation is still limited due to the restricted availability of agricultural area and the increased use of land in actions for tourism and building construction. Based on the foregoing, it is suggested that possible regions and/or crop cultivation practices for high quality and added value MAPs must be evaluated [27], so that farmers can switch to these crops and create profitable and successful farms. Due to the rising worldwide demand for high-value MAPs, Cyprus, which has a lengthy history of MAPs cultivation and use, might become a key location for producing and trading high-quality raw materials of MAPs, even to the more industrialized countries for additional processing.

Salvia spp. is the largest and the most well-known genus in the Lamiaceae family, with over 900 medicinal and ornamental species distributed all over the world [28]. The genus Mentha spp. belongs also to the Lamiaceae family and includes 25 to 30 species that 
grow in the temperate regions of Europe, Australia, Africa, Asia, and North America [29]. The genus Sideritis L. (Lamiaceae) comprises more than 150 species that can be found in the Mediterranean, the Balkans and the Iberian Peninsula [30]. Several researchers have studied the correlation between total phenolics and/or phenolic compounds content and antioxidant capacity of different MAPs products, such as infusions, decoctions and EOs [14,31-33]. As an example, the antioxidant capacity and phenolics components in ten Serbia MAPs demonstrated a positive correlation between phenolics and tannins, as well as a proportional rise in antioxidants with total phenolics [33]. The correlations between the primary EOs constituents and the phenolics content and the antioxidant capacity of leaves from one hand, and the plant mineral content on the other hand, have received little attention [34]. As a result, in order to enrich the existing knowledge, the aim of this study was (i) to compare selected MAPs grown in Cyprus under various environmental conditions (altitudes; mountainous and plain areas) (ii) to examine the effects of the season on three MAPs, in order to uncover possible correlations between leaf antioxidant activity and their mineral content and their essential oil (EO) yield and composition. The plant species selected for this study were chosen based on their popularity and their wide variety of applications.

\section{Materials and Methods}

\subsection{Plant Material and Growing Conditions}

The medicinal and aromatic plants used in the present study were sage (Salvia officinalis L.), sideritis (Sideritis perfoliata L. subsp. perfoliata) and spearmint (Mentha spicata L.) and the parts used from each species are presented in Table 1 . The cultivated plants were initially purchased for both studied areas (plain and mountainous) as seedlings for sage and sideritis and as cuttings for spearmint by the Cypriot National Agricultural Department, and the harvested plantations were aged of 4-5 years for sage and sideritis and of 1-2 years for spearmint.

In the current study, two areas with different climatic conditions (for simplicity the term altitude will be used, given the differences in the microclimates as indicated by Kofidis and Bosabalidis [11]) were selected, the mountainous area of "Gerasa" village $\left(34^{\circ} 49^{\prime} 37.59^{\prime \prime} \mathrm{N} ; 33^{\circ} 0^{\prime} 26.46^{\prime \prime} \mathrm{E}\right)$ and the plain area of Limassol ("Akrotiri"; $34^{\circ} 38^{\prime} 1.63^{\prime \prime} \mathrm{N}$; $\left.32^{\circ} 56^{\prime} 3.62^{\prime \prime} \mathrm{E}\right)$. The village area of "Gerasa" is located at $623 \mathrm{~m}$ above sea level. The climate there is characterized by low temperatures, with approx. four freezing days and the lowest recorded temperature of soil surface during winter down to $-8.1{ }^{\circ} \mathrm{C}$ (February). The soil in the area has sand/silk texture, $\mathrm{pH}$ of 8.04, electrical conductivity (EC) of $0.52 \mathrm{mS} \mathrm{cm}^{-1}$, and appeared poor in organic matter $(<0.5 \%)$ and nitrogen- $0.26 \mathrm{~g} \mathrm{~kg}^{-1}$. On the contrary, the plain area of "Akrotiri" is a seaside area, located at $2 \mathrm{~m}$ above sea level, with mild winter and dry-hot summer. The physicochemical characteristics of the soil were: sand/clay loam

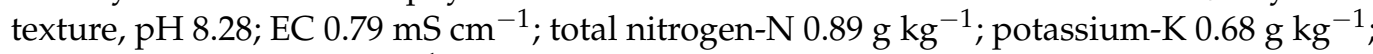
phosphorus-P $0.017 \mathrm{~g} \mathrm{~kg}^{-1}$ and organic matter $2.88 \%$. Detailed climatic conditions of the selected areas are described in supplementary material (Figure S1); mean daytime temperatures were averaged at $16.8^{\circ} \mathrm{C}$ and $19.9{ }^{\circ} \mathrm{C}$, and air humidity averaged in $56.3 \%$ and $66.1 \%$, for mountain and plain areas, respectively. Maximum daytime temperature reached $42.4^{\circ} \mathrm{C}$ and $40.2{ }^{\circ} \mathrm{C}$ with $9.3-13.7 \mathrm{~mm}$ and $0 \mathrm{~mm}$ rainfall for mountain and plain areas, respectively, during the mid-summer period.

Both plantations for plain and mountainous areas were farming by the same producer. Plant species from an organic plantation were harvested from the plain area. Common cultivation practices were applied, plants were frequently irrigated ( $\sim$ weekly/biweekly during the growing period) based on the plant needs and common fertilizers and crop protection means were applied as shown in Table S1. Mountain species were harvested also from an organic plantation, and plants were grown under commercial cultivation practices, but plants received conservation practices (periodical irrigation and few fertilizers or pesticides' application).

Plant tissue (nine samples/area/species) of the above ground parts (leaves/stems or leaves/stems/flowers) (see also Table 1) was collected in four sampling periods, namely 
summer (June 2018), autumn (October 2018), winter (January 2019) and spring (April 2019), and transferred within an hour to the laboratory. Each sample was divided in two batch samples: one batch was dried at air-forced oven at $42{ }^{\circ} \mathrm{C}$ until constant weight for approximately 3-4 days and used for the essential oil extraction (see Section 2.4) and mineral content determination (see Section 2.3), while the other batch was stored at $-20{ }^{\circ} \mathrm{C}$ for the chemical analyses described in Section 2.2.

Table 1. Lamiaceae family plant species and material used.

\begin{tabular}{|c|c|c|c|}
\hline Common Name & Latin Name & Plant Material & $\begin{array}{c}\text { Reported Medicinal } \\
\text { Properties/Indications }\end{array}$ \\
\hline Spearmint & Mentha spicata $\mathrm{L}$. & $\begin{array}{l}\text { Stem/ } \\
\text { leaves }\end{array}$ & $\begin{array}{l}\text { Anti-inflammatory, sedative, antimicrobial, } \\
\text { antioxidant, carminative, antispasmodic, diuretic, } \\
\text { insecticidal, vasoconstrictor, } \\
\text { decongestant }[29,35,36] \text {. }\end{array}$ \\
\hline Sideritis & Sideritis perfoliata L. subsp. perfoliata & $\begin{array}{l}\text { Stem/ } \\
\text { Leaves / } \\
\text { flowers }\end{array}$ & $\begin{array}{l}\text { Anti-inflammatory, antimicrobial, vulnerary, } \\
\text { antioxidant, antispasmodic, analgesic, stomachic, } \\
\text { carminative, anti-rheumatic, anti-ulcerative, } \\
\text { digestive, vasoprotective }[27,30,37]\end{array}$ \\
\hline Sage & Salvia officinalis L. & $\begin{array}{l}\text { Stem/ } \\
\text { leaves }\end{array}$ & $\begin{array}{l}\text { Antibacterial, antifungal, anticancer, antiviral, } \\
\text { antidiabetic, antimutagenic, antiprotozoal, } \\
\text { antidementia, antioxidant, anti-inflammatory, } \\
\text { anti-nociceptive, antidementia, antiseptic, } \\
\text { antispasmoic, astringent, antihidrotic, } \\
\text { hypoglycemic, and hypolipidemic effects }[12,38,39]\end{array}$ \\
\hline
\end{tabular}

\subsection{Total Phenols Content and Antioxidant and Reducing Activity}

Four samples $(0.5 \mathrm{~g})$ of freshly cut plants (pooled by three individual plants/sample) from each treatment (four seasons or two altitudes) were milled with $10 \mathrm{~mL}$ methanol $(50 \% v / v)$ [40]. The extracts were centrifuged for $30 \mathrm{~min}$ at $4000 \times g$ at $4{ }^{\circ} \mathrm{C}$ (Sigma 3-18 K, Sigma Laboratory Centrifuge, Taufkirchen, Germany), and the supernatant was transferred to a $15 \mathrm{~mL}$ falcon tube for the determination of total phenol content and total antioxidant activity.

The total phenol content of the methanolic extracts was determined by using the Folin-Ciocalteu reagent (Merck), based on previous described procedure [40] and results were expressed as gallic acid equivalents (mg GAE per $\mathrm{g}$ of fresh weight). The antioxidant activity of the methanolic plant extracts was determined by using the assays of ferric reducing antioxidant power (FRAP), as previously described by Chrysargyris et al. [41], as well as the 2,2'-azino-bis(3-ethylbenzothiazoline-6-sulphonic acid) (ABTS) assay according to the methodology described by Woidjylo et al. [42] by using standard solution of trolox (( \pm )-6-Hydroxy-2,5,7,8-tetramethylchromane-2-carboxylic acid). The results were expressed as mg trolox $\mathrm{g}^{-1} \mathrm{Fw}$.

\subsection{Mineral Content}

Dried tissue $(0.5 \mathrm{~g})$ from the aerial plant parts from each treatment (four biological replications; each replication was a pool of two individual plants) was ashed $\left(490^{\circ} \mathrm{C}\right)$ and acid-digested $(2 \mathrm{~N} \mathrm{HCl})$ for mineral extraction. Sodium $(\mathrm{Na})$ and potassium $(\mathrm{K})$ were determined with flame photometry (Lasany Model 1832, Lasany International, Haryana, India), phosphorus (P) with the molybdate/vanadate method (yellow method) by spectrophotometry (Multiskan GO, Thermo Fischer Scientific, Massachusetts, MA, USA), nitrogen (N) with the Kjeldahl method (BUCHI, Digest automat K-439 and Distillation Kjelflex K-360, Flawil, Switzerland) and calcium (Ca), magnesium $(\mathrm{Mg})$, iron $(\mathrm{Fe})$, copper $(\mathrm{Cu})$ and zinc (Zn) by an atomic absorption spectrophotometer (PG Instruments AA500 FG, Leicestershire, UK) following the method of Chrysargyris et al. [43]. Plant minerals content was expressed in $\mathrm{g} \mathrm{kg}^{-1}$ and $\mathrm{mg} \mathrm{kg}^{-1}$ of dry weight for macronutrients and micronutrients, respectively. 


\subsection{Essential Oil Extraction and Gas Chromatography/Mass Spectrometry Analysis}

The essential oils were extracted via hydrodistillation using a Clevenger apparatus according to the protocol previously described by the authors [44]. In brief, dried aerial parts (50-60 g for each treatment) of the plants were used for the EO extraction, which lasted for $3 \mathrm{~h}$, while each treatment was replicated three times. The essential oil (dried over anhydrous sodium sulphate) yield was measured and calculated as percentage of oil per dry weight $(\mathrm{dw})$ [44]. The obtained EOs were kept in amber glass bottles at $-20^{\circ} \mathrm{C}$ until GC/MS analysis was performed.

Analytical gas chromatography was carried out with a Shimadzu GC2010 gas chromatograph interfaced Shimadzu GC/MS QP2010 plus mass spectrometer based on the protocol previously described by the authors [44]. An aliquot of $2 \mu \mathrm{L}$ of each sample was injected in a split mode (split ratio 20:1) into the gas chromatograph fitted with a ZB-5 column (Zebron, Phenomenex, Torrance, CA, USA) coated with $5 \%$ pheny- $95 \%$ dimethylpolysiloxane with film thickness of $0.25 \mu \mathrm{m}$, length of $30.0 \mathrm{~m}$ and a diameter of $0.25 \mathrm{~mm}$. The flow of the carrier gas (helium) was $1.03 \mathrm{~mL} \mathrm{~min}^{-1}$. The injector temperature was set at $230^{\circ} \mathrm{C}$. Electron impact mass spectra with ionization energy of $70 \mathrm{eV}$ was recorded at the $35-400 \mathrm{~m} \mathrm{z}^{-1}$. The column temperature was programmed to rise from $60^{\circ} \mathrm{C}$ to $240{ }^{\circ} \mathrm{C}$ at a rate of $5{ }^{\circ} \mathrm{C} \mathrm{min}^{-1}$, with a $5 \mathrm{~min}$ hold at $240^{\circ} \mathrm{C}$. The solution of standard alkanes mixture (C8-C20) was also analyzed using the above conditions.

Components were identified through the comparison of their retention indices relative to n-alkanes (C8-C20) with those of the literature or with those of authentic compounds when available. Further identification of compounds was carried out by matching the recorded mass spectra with those stored in the NIST08 mass spectral library of the GC-MS data system and published mass spectra in the literature [44]. The percentage of individual compounds was based on peak area normalization without using correction factors.

\subsection{Statistical Methods}

A three factor (Species, Seasons and Environmental Conditions-namely Altitude) factorial study was carried out. The statistical treatment of the results was carried out using a three-way analysis of variance (ANOVA) by using the IBM SPSS v.22 software for Windows (IBM Corp., Armonk, NY, USA). Duncan's Multiple Range Test was used for the comparison of means in the cases where the effect of Species, Seasons and Altitude and their relevant interactions were significant. Mean values are presented as treatment mean \pm SE of four biological measurements $(n=4)$ for antioxidants and mineral content and of three biological measurements $(n=3)$ for essential oils analysis. The correlation coefficients between mountainous/plain species and seasons and their antioxidant capacity and essential oil components were also determined. Pairwise metabolite effect correlations were calculated by Pearson's correlation test using the R program.

\section{Results and Discussion}

\subsection{Total Phenols and Antioxidant Capacity}

Phenolic molecules are the most important classes of natural antioxidants, and they are highly correlated with the antioxidant activity in plant tissues [45]. In this study, we determined whether the content of total phenols and the antioxidant activity of three MAP species were affected by the season [46] and by the altitude, as it has been previously reported $[32,34]$. The light intensity and photoperiod have different effects on the accumulation of plant secondary metabolites in different plants [2]. Table 2 shows the impact of environmental conditions-altitude (mountain versus plain), season (summer, autumn, winter and spring) and plant species, on the total phenols content, antioxidant activity, essential oil yield and mineral content of the examined MAP species. The three-way ANOVA revealed that all the examined parameters were affected significantly $(p<0.001, p<0.01)$ by the season, significantly $(p<0.001, p<0.05)$ by the altitude (except for FRAP, leaf $\mathrm{Cu}$ and $\mathrm{Zn}$ content), and significantly $(p<0.001)$ by the species (except for leaf $\mathrm{Cu}$ content). The interaction between the examined factors (seasons, species and altitude) for all the 
investigated parameters revealed that season * altitude and altitude * species did not affect leaf Zn content (including ABTS levels for the latter). Moreover, the interaction of season * species and the season * altitude ${ }^{*}$ species significantly affected all the other parameters.

Table 2. Effects of altitude (mountain vs. plain) and season (summer, autumn, winter and spring) on the content of total phenols (mg GAE $\mathrm{g}^{-1} \mathrm{Fw}$ ), antioxidant and reducing activity (FRAP and ABTS, $\mathrm{mg}$ trolox $\mathrm{g}^{-1} \mathrm{Fw}$ ), macronutrient ( $\mathrm{g} \mathrm{kg}{ }^{-1}$ ) and micronutrient content $\left(\mathrm{mg} \mathrm{kg}^{-1}\right)$ content and essential oil (EO) yield (\%) in selected medicinal plant species.

\begin{tabular}{|c|c|c|c|c|c|c|c|}
\hline Factors & Season & Altitude & Species & $\begin{array}{l}\text { Season* } \\
\text { Altitude }\end{array}$ & $\begin{array}{l}\text { Season* } \\
\text { Species }\end{array}$ & $\begin{array}{l}\text { Altitude * } \\
\text { Species }\end{array}$ & $\begin{array}{c}\text { Season * Altitude * } \\
\text { Species }\end{array}$ \\
\hline Phenols (mg GAE g ${ }^{-1}$ ) & $* * *$ & * & $* * *$ & $* * *$ & $* * *$ & $* * *$ & $* * *$ \\
\hline FRAP (mg trolox $\left.\mathrm{g}^{-1}\right)$ & $* * *$ & ns & $* * *$ & $* * *$ & $* * *$ & $* * *$ & $* * *$ \\
\hline ABTS (mg trolox $\mathrm{g}^{-1}$ ) & $* * *$ & $* * *$ & $* * *$ & $* * *$ & $* * *$ & ns & $* * *$ \\
\hline $\mathrm{N}\left(\mathrm{g} \mathrm{kg}^{-1}\right)$ & $* * *$ & $* * *$ & $* * *$ & $* * *$ & $* * *$ & $* * *$ & $* * *$ \\
\hline $\mathrm{K}\left(\mathrm{g} \mathrm{kg}^{-1}\right)$ & $* * *$ & $* * *$ & $* * *$ & $* * *$ & $* * *$ & $* * *$ & $* * *$ \\
\hline $\mathrm{P}\left(\mathrm{g} \mathrm{kg}^{-1}\right)$ & $* * *$ & $* * *$ & $* * *$ & $* * *$ & $* * *$ & $* * *$ & $* * *$ \\
\hline $\mathrm{Na}\left(\mathrm{g} \mathrm{kg}^{-1}\right)$ & $* * *$ & $* * *$ & $* * *$ & $* * *$ & $* * *$ & $* * *$ & $* * *$ \\
\hline $\mathrm{Ca}\left(\mathrm{g} \mathrm{kg}^{-1}\right)$ & $* * *$ & $* * *$ & $* * *$ & $* * *$ & $* * *$ & $* * *$ & $* * *$ \\
\hline $\operatorname{Mg}\left(\mathrm{g} \mathrm{kg}^{-1}\right)$ & $* * *$ & $* * *$ & $* * *$ & $* * *$ & $* * *$ & $* * *$ & $* * *$ \\
\hline $\mathrm{Fe}\left(\mathrm{mg} \mathrm{kg}^{-1}\right)$ & $* * *$ & $* * *$ & $* * *$ & $* * *$ & $* * *$ & ** & $* * *$ \\
\hline $\mathrm{Zn}\left(\mathrm{mg} \mathrm{kg}^{-1}\right)$ & $* * *$ & ns & $* * *$ & ns & $* * *$ & ns & $* * *$ \\
\hline $\mathrm{Cu}\left(\mathrm{mg} \mathrm{kg} \mathrm{g}^{-1}\right)$ & $* *$ & ns & ns & * & $* * *$ & * & $* *$ \\
\hline $\mathrm{EO}(\%)$ & $* * *$ & $* * *$ & $* * *$ & $* * *$ & $* * *$ & $* * *$ & $* * *$ \\
\hline
\end{tabular}

$\mathrm{ns},{ }^{*}{ }^{* *}$, and ${ }^{* * *}$ indicate non-significant or significant differences at $p<0.05, p<0.01$, and $p<0.001$, respectively, following a threeway ANOVA.

Eco-geographical factors do affect the biosynthesis of secondary metabolites; the effect of collection region on total phenols and antioxidants has previously been reported for Salvia argentea, Salvia officinalis, and Salvia verbenaca $[31,47,48]$. Altitude and seasonal collection has also been found to affect the levels of phenolics and antioxidant capacity of plant species $[34,46,48]$ among others. Different environmental factors, such as $\mathrm{CO}_{2}$ levels, water availability, temperature and sun radiation, can influence secondary metabolism and stimulate the production of bioactive chemicals [49]. In general, season affected antioxidant status of the examined species. Therefore, plants presented higher total phenols $\left(18.18 \pm 1.98 \mathrm{mg} \mathrm{GAE}^{-1} \mathrm{Fw}\right)$ and antioxidant levels based on FRAP $\left(35.31 \pm 2.70 \mathrm{mg}\right.$ trolox $\left.\mathrm{g}^{-1} \mathrm{Fw}\right)$ and ABTS $\left(24.93 \pm 2.76 \mathrm{mg}\right.$ trolox $\left.\mathrm{g}^{-1} \mathrm{Fw}\right)$ assays during winter (including spring season for the ABTS levels) in comparison to plants harvested during summer period (Table 3). However, altitude did not affect phenols and the level of antioxidants of the examined species. Interestingly, when comparing all the species regardless the season and the altitude, total phenols levels were higher in sage $\left(21.53 \pm 1.25 \mathrm{mg} \mathrm{GAE} \mathrm{g}^{-1} \mathrm{Fw}\right)$, in comparison to sideritis and spearmint. Similarly, sage revealed the highest antioxidant activity for both FRAP and ABTS assays (34.97 \pm 3.08 and $27.94 \pm 1.77 \mathrm{mg}$ trolox $\mathrm{g}^{-1} \mathrm{Fw}$, respectively), followed by spearmint and then by sideritis. Sideritis also revealed almost half the antioxidant capacity compared to sage. Previous research on the effects of season collection on the total phenols levels in Rosmarinus officinalis revealed that during summer, plants had the lowest total phenolics content [46], which is consistent with the current outcomes.

On the other hand, when evaluating the combined effect of the examined factors, mountainous sage plants presented the highest content of total phenols (ranging from 28.13-32.85 $\mathrm{mg} \mathrm{GAE} \mathrm{g}^{-1} \mathrm{Fw}$ ) during autumn-winter with the highest antioxidant capacity (FRAP; $67.97 \pm 4.02 \mathrm{mg} \pm$ trolox $\mathrm{g}^{-1} \mathrm{Fw}$ and ABTS; $46.29 \pm 1.91 \mathrm{mg}^{\text {trolox }} \mathrm{g}^{-1} \mathrm{Fw}$, respectively) pointed during winter (Table 3 ). The antioxidant capacity of plants is inversely

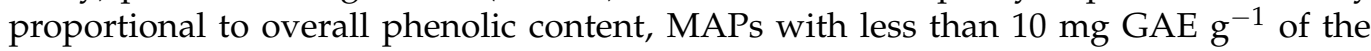
extract having the lowest antioxidant capacity [33], a finding that was also observed in the present study in the case of sideritis (both altitudes) during summer, plain sideritis 
during autumn, mountainous sideritis during winter and mountainous spearmint during summer (Table 3).

Table 3. Effects of altitude (mountain vs. plain) and season (summer, autumn, winter and spring) on the content of total phenols (mg GAE g ${ }^{-1} \mathrm{Fw}$ ), antioxidant and reducing activity (FRAP and ABTS, $\mathrm{mg}$ trolox $\mathrm{g}^{-1} \mathrm{Fw}$ ) and essential oil (EO) yield (\%) in selected medicinal plant species.

\begin{tabular}{|c|c|c|c|c|c|c|}
\hline Species & Season & Altitude & Total Phenols & FRAP & ABTS & EO \\
\hline Sage & & & $21.53 \pm 1.25 \mathrm{~A}$ & $34.97 \pm 3.08 \mathrm{~A}$ & $27.94 \pm 1.77 \mathrm{~A}$ & $2.56 \pm 0.23 \mathrm{~A}$ \\
\hline Sideritis & & & $11.27 \pm 1.13 \mathrm{~B}$ & $16.73 \pm 1.92 \mathrm{~B}$ & $14.00 \pm 1.95 \mathrm{~B}$ & $0.34 \pm 0.04 \mathrm{C}$ \\
\hline Spearmint & & & $14.84 \pm 0.91 \mathrm{~B}$ & $30.66 \pm 2.67 \mathrm{~A}$ & $18.26 \pm 1.85 \mathrm{~B}$ & $1.25 \pm 0.16 \mathrm{~B}$ \\
\hline \multirow[t]{9}{*}{ Total mean } & & & $15.88 \pm 0.81$ & $27.45 \pm 1.74$ & $20.07 \pm 1.26$ & $1.38 \pm 0.14$ \\
\hline & Summer & & $12.19 \pm 1.37 \mathrm{~B}$ & $20.16 \pm 3.07 \mathrm{~B}$ & $13.29 \pm 2.16 \mathrm{~B}$ & $1.92 \pm 0.31 \mathrm{~A}$ \\
\hline & Autumn & & $15.32 \pm 1.80 \mathrm{AB}$ & $22.57 \pm 2.70 \mathrm{~B}$ & $16.95 \pm 2.32 \mathrm{AB}$ & $1.93 \pm 0.34 \mathrm{~A}$ \\
\hline & Winter & & $18.18 \pm 1.98 \mathrm{~A}$ & $35.31 \pm 4.20 \mathrm{~A}$ & $24.93 \pm 2.76 \mathrm{~A}$ & $0.67 \pm 0.19 \mathrm{~B}$ \\
\hline & Spring & & $17.82 \pm 0.69 \mathrm{AB}$ & $31.75 \pm 2.70 \mathrm{AB}$ & $25.11 \pm 1.68 \mathrm{~A}$ & $1.02 \pm 0.14 \mathrm{AB}$ \\
\hline & Total mean & & $15.88 \pm 0.81$ & $27.45 \pm 1.74$ & $20.07 \pm 1.26$ & $1.38 \pm 0.14$ \\
\hline & & Mountain & $16.37 \pm 1.32 \mathrm{~A}$ & $27.37 \pm 2.79 \mathrm{~A}$ & $21.89 \pm 1.97 \mathrm{~A}$ & $1.13 \pm 0.14 \mathrm{~A}$ \\
\hline & & Plain & $15.39 \pm 0.93 \mathrm{~A}$ & $27.53 \pm 2.14 \mathrm{~A}$ & $18.24 \pm 1.55 \mathrm{~A}$ & $1.64 \pm 0.24 \mathrm{~A}$ \\
\hline & & Total mean & $15.88 \pm 0.81$ & $27.45 \pm 1.74$ & $20.07 \pm 1.26$ & $1.38 \pm 0.14$ \\
\hline \multirow[t]{8}{*}{ Sage } & Summer & Mountain & $18.86 \pm 0.25 b^{\mathrm{Y}}$ & $21.04 \pm 0.56$ efghi & $21.17 \pm 1.09$ defgh & $2.78 \pm 0.11 \mathrm{c}$ \\
\hline & & Plain & $18.18 \pm 2.01 \mathrm{bc}$ & $30.15 \pm 5.92$ cdef & $28.65 \pm 1.73 \mathrm{bcd}$ & $3.85 \pm 0.15 b$ \\
\hline & Autumn & Mountain & $28.13 \pm 1.22 \mathrm{a}$ & $38.63 \pm 3.66 \mathrm{bcd}$ & $32.96 \pm 2.66 b c$ & $2.37 \pm 0.09 \mathrm{~cd}$ \\
\hline & & Plain & $19.28 \pm 1.61 \mathrm{bc}$ & $29.83 \pm 2.13 \mathrm{cdef}$ & $22.61 \pm 2.20$ cdefg & $4.76 \pm 0.19 a$ \\
\hline & Winter & Mountain & $32.85 \pm 1.56 \mathrm{a}$ & $67.97 \pm 4.02 \mathrm{a}$ & $46.29 \pm 1.91 \mathrm{a}$ & $1.46 \pm 0.05 \mathrm{fg}$ \\
\hline & & Plain & $22.92 \pm 0.88 b$ & $41.51 \pm 0.56 \mathrm{bc}$ & $28.62 \pm 1.72 \mathrm{bcd}$ & $2.05 \pm 0.08 \mathrm{de}$ \\
\hline & Spring & Mountain & $14.83 \pm 0.52$ cde & $23.15 \pm 1.83 \mathrm{efgh}$ & $22.33 \pm 2.35$ cdefg & $1.48 \pm 0.06 \mathrm{fg}$ \\
\hline & & Plain & $17.17 \pm 1.25 \mathrm{~cd}$ & $27.47 \pm 2.93 \mathrm{def}$ & $20.94 \pm 1.91$ defgh & $1.75 \pm 0.07 \mathrm{ef}$ \\
\hline \multirow[t]{8}{*}{ Sideritis } & Summer & Mountain & $7.08 \pm 0.14 \mathrm{hi}$ & $7.87 \pm 0.24 j$ & $6.31 \pm 0.26 \mathrm{ij}$ & $0.35 \pm 0.01$ hijk \\
\hline & & Plain & $5.70 \pm 0.54 \mathrm{i}$ & $9.39 \pm 0.90 \mathrm{ij}$ & $3.10 \pm 0.18 \mathrm{j}$ & $0.49 \pm 0.01 \mathrm{hij}$ \\
\hline & Autumn & Mountain & $12.80 \pm 1.59 \mathrm{def}$ & $20.58 \pm 2.01 \mathrm{fghi}$ & $17.14 \pm 1.31$ defghi & $0.67 \pm 0.02 \mathrm{~h}$ \\
\hline & & Plain & $4.31 \pm 1.01 \mathrm{i}$ & $5.27 \pm 1.38 \mathrm{j}$ & $3.65 \pm 1.10 \mathrm{j}$ & $0.54 \pm 0.02 \mathrm{hi}$ \\
\hline & Winter & Mountain & $8.22 \pm 0.45 \mathrm{fghi}$ & $11.78 \pm 0.62$ hij & $11.73 \pm 1.57$ ghij & $0.09 \pm 0.00 \mathrm{jk}$ \\
\hline & & Plain & $19.12 \pm 0.39 \mathrm{bc}$ & $32.63 \pm 1.37$ cde & $26.00 \pm 2.09$ bcde & $0.12 \pm 0.00 \mathrm{jk}$ \\
\hline & Spring & Mountain & $16.31 \pm 0.38$ cde & $21.34 \pm 1.18$ efghi & $24.89 \pm 6.50$ bcdef & $0.21 \pm 0.01 \mathrm{ijk}$ \\
\hline & & Plain & $16.65 \pm 1.34$ cde & $24.97 \pm 2.49 \mathrm{efg}$ & $19.23 \pm 1.70$ defgh & $0.29 \pm 0.01$ hijk \\
\hline \multirow[t]{8}{*}{ Spearmint } & Summer & Mountain & $7.61 \pm 0.15 \mathrm{ghi}$ & $11.54 \pm 0.39 \mathrm{hij}$ & $9.96 \pm 0.92$ ghij & $1.39 \pm 0.05 \mathrm{fg}$ \\
\hline & & Plain & $15.75 \pm 0.44$ cde & $40.99 \pm 2.66 \mathrm{bc}$ & $10.55 \pm 0.47 \mathrm{hij}$ & $2.65 \pm 0.10 c$ \\
\hline & Autumn & Mountain & $12.66 \pm 0.86 \mathrm{defg}$ & $26.28 \pm 1.88 \mathrm{efg}$ & $14.05 \pm 2.42$ fghij & $1.35 \pm 0.05 \mathrm{fg}$ \\
\hline & & Plain & $14.73 \pm 0.31 \mathrm{def}$ & $14.84 \pm 1.67$ ghij & $11.30 \pm 1.57$ ghij & $1.90 \pm 0.07 \mathrm{e}^{\circ}$ \\
\hline & Winter & Mountain & $14.33 \pm 0.21 \mathrm{def}$ & $31.65 \pm 0.81$ cdef & $20.48 \pm 2.00$ defgh & $0.23 \pm 0.01 \mathrm{ijk}$ \\
\hline & & Plain & $11.67 \pm 0.70 \mathrm{efgh}$ & $26.35 \pm 1.14 \mathrm{efg}$ & $16.46 \pm 1.92$ efghi & $0.07 \pm 0.00 \mathrm{k}$ \\
\hline & Spring & Mountain & $22.82 \pm 0.59 \mathrm{~b}$ & $46.65 \pm 1.05 \mathrm{~b}$ & $35.44 \pm 1.11 \mathrm{ab}$ & $1.13 \pm 0.04 \mathrm{~g}$ \\
\hline & & Plain & $19.18 \pm 0.54 \mathrm{bc}$ & $46.95 \pm 1.31 b$ & $27.85 \pm 1.49$ bcde & $1.29 \pm 0.05 \mathrm{~g}$ \\
\hline
\end{tabular}

${ }^{Y}$ values (means $\pm \mathrm{SE}, n=4$ ) in columns corresponding to the main factors (Altitude, Seasons and Species) followed by the same uppercase letter, and values corresponding to the interaction of the main factors (Altitude, Seasons and Species), which are followed by the same lowercase letter, are not significantly different, $p<0.05$.

\subsection{Mineral Content}

The impact of season and altitude on nutrient accumulation in sage, sideritis and spearmint plants is presented in Table 4 . In general, season affected $\mathrm{P}, \mathrm{Na}$ and Fe accumulation in plants, as during winter, plants accumulated more $\mathrm{P}\left(3.06 \pm 0.25 \mathrm{~g} \mathrm{~kg}^{-1}\right)$ and $\mathrm{Na}\left(1.40 \pm 0.30 \mathrm{~g} \mathrm{~kg}^{-1}\right)$, while Fe was accumulated more in summer-autumn and winter period, and ranged from $336.07 \pm 9.77$ to $346.38 \pm 16.07 \mathrm{mg} \mathrm{kg}^{-1}$. The accumulation of $\mathrm{N}, \mathrm{K}, \mathrm{Ca}, \mathrm{Mg}, \mathrm{Zn}$ and $\mathrm{Cu}$ was not affected by the season. Regarding altitude, plain plants accumulated more $\mathrm{N}, \mathrm{K}, \mathrm{Na}$ and $\mathrm{Ca}$ comparing to the mountainous plants, while the latter had higher (up to 8.5\%) levels of Fe. Among the exam- 
ined MAPs, spearmint accumulated more $\mathrm{N}\left(27.96 \pm 1.16 \mathrm{~g} \mathrm{~kg}^{-1}\right), \mathrm{P}\left(3.07 \pm 0.18 \mathrm{~g} \mathrm{~kg}^{-1}\right)$, $\mathrm{Na}\left(1.22 \pm 0.22 \mathrm{~g} \mathrm{~kg}^{-1}\right)$ and $\mathrm{Mg}\left(11.42 \pm 0.43 \mathrm{~g} \mathrm{~kg}^{-1}\right)$, while increased $\mathrm{K}$ levels were found in spearmint $\left(20.77 \pm 1.10 \mathrm{~g} \mathrm{~kg}^{-1}\right)$ and sideritis $\left(18.65 \pm 0.81 \mathrm{~g} \mathrm{~kg}^{-1}\right)$ plants. Additionally, sideritis plants had high Fe content $\left(345.29 \pm 17.41 \mathrm{mg} \mathrm{kg}^{-1}\right)$, while sage had high $\mathrm{Zn}$ content $\left(42.76 \pm 2.43 \mathrm{mg} \mathrm{kg}^{-1}\right)$.

On the other hand, when evaluating the combined effect of the examined factors, during summer, mountainous spearmint accumulated more $\mathrm{Mg}$ and $\mathrm{Cu}$, while mountainous sage accumulated more $\mathrm{P}$, and plain sideritis had the highest Fe content. During autumn, plain sideritis had the highest $\mathrm{K}$ content. During winter, mountainous spearmint presented the highest content on $\mathrm{N}$ and $\mathrm{K}$ while plain spearmint and sage accumulated more $\mathrm{Na}$ and $\mathrm{Zn}$, respectively. Moreover, during winter, plain spearmint revealed the highest content of P. During spring, mountainous sage revealed the highest $\mathrm{Ca}$ content and plain spearmint the highest K content (Table 4). Nutrient levels play an important role in the growth and development of the plants, while minerals are mainly uptaken by the plants from soil, or from the application of fertilizers, through soil or foliar. The level of nutrients inside plant tissue may not only affect plant growth but also has effects on the production and the concentration of secondary metabolites as antioxidants and essential oils. The concentration of Germacrene D in basil EO, for example, is impacted by the rate of applied $\mathrm{N}$ and the interaction between $\mathrm{N}$ and $\mathrm{K}$, but not by the rate of applied K [50]. Rosemary plant growth and oil yield were significantly dependent on $\mathrm{N}$ and $\mathrm{K}$ application [51].

\subsection{Essential Oil Yield and Composition}

Several aromatic plants have been documented to have their oil content and composition influenced by weather variables such as ambient temperature and rainfall [52]. It should be noted, however, that the time when the more EO is obtained may not be the time when the oil has the greatest production of the chemical constituent(s) of interest [53]. Table 2 presents the effects of environmental conditions-altitude (mountain versus plain), season (summer, autumn, winter and spring) and species on the EOs yield of the examined MAP species. The three-way ANOVA reveled that EOs' yield was affected by season $(p<0.01)$, altitude $(p<0.01)$, species $(p<0.001)$ and by the interaction of the three factors $(p<0.001)$. Both summer $(1.93 \pm 0.32 \%)$ and autumn $(1.93 \pm 0.34 \%)$ seasons resulted in higher EO yield compared to winter $(0.67 \pm 0.19 \%)$ when the species and altitude factors were not considered. In general, winter harvest significantly reduced EO yield in Cymbopogon winterianus independently of the actual harvest time [13]. From February to July, the EO content of sage (S. officinalis) plants grown in Italy was increased by more than two-fold [54]. During autumn-winter in Cyprus, rainfall was ranged from 73.1-142.6 mm and 52.1-94.5 mm from November till February, in mountainous and plain areas, respectively (Figure S1). Indeed, rainfall exerts effects on the vegetative stage of the plants and can directly influence the production of EO; however, during winter low temperatures slowed down the production of the secondary metabolites, decreasing the EO yield. Similar observations have been reported previously, when harvesting took place in autumn (end October) in the same island, but in different altitudes [34]. However, other researchers reported that low temperature was positively correlated to the EO yield of R. officinalis in Brazil [46]. Since the production and composition of EO and extracts of plants depend on genetics, environmental conditions and plant part [55], this can explain a good portion of the discrepancy among the results obtained by different studies. 


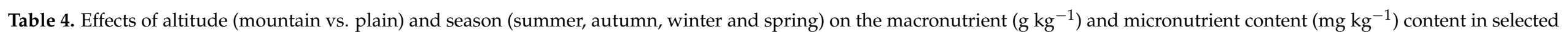
medicinal plant species.

\begin{tabular}{|c|c|c|c|c|c|c|c|c|c|c|c|}
\hline Species & Season & Altitude & $\mathbf{N}$ & K & $\mathbf{P}$ & $\mathrm{Na}$ & $\mathrm{Ca}$ & Mg & $\mathrm{Fe}$ & $\mathrm{Zn}$ & $\mathrm{Cu}$ \\
\hline Sage & & & $15.71 \pm 1.09 \mathrm{~B}$ & $15.33 \pm 0.85 \mathrm{~B}$ & $2.25 \pm 0.27 \mathrm{~B}$ & $0.74 \pm 0.08 \mathrm{AB}$ & $8.22 \pm 0.64 \mathrm{~A}$ & $8.71 \pm 0.28 \mathrm{~B}$ & $335.56 \pm 10.39 \mathrm{AB}$ & $42.76 \pm 2.43 \mathrm{~A}$ & $41.48 \pm 1.78 \mathrm{~A}$ \\
\hline Sideritis & & & $17.87 \pm 0.53 \mathrm{~B}$ & $18.65 \pm 0.81 \mathrm{~A}$ & $2.44 \pm 0.13 \mathrm{AB}$ & $0.46 \pm 0.08 \mathrm{~B}$ & $7.37 \pm 0.47 \mathrm{~A}$ & $8.07 \pm 0.56 \mathrm{~B}$ & $345.29 \pm 17.41 \mathrm{~A}$ & $12.76 \pm 1.08 \mathrm{~B}$ & $42.45 \pm 2.82 \mathrm{~A}$ \\
\hline Spearmint & & & $27.96 \pm 1.16 \mathrm{~A}$ & $20.77 \pm 1.10 \mathrm{~A}$ & $3.07 \pm 0.18 \mathrm{~A}$ & $1.22 \pm 0.22 \mathrm{~A}$ & $8.06 \pm 0.17 \mathrm{~A}$ & $11.42 \pm 0.43 \mathrm{~A}$ & $297.05 \pm 9.09 \mathrm{~B}$ & $14.86 \pm 1.54 \mathrm{~B}$ & $41.81 \pm 3.72 \mathrm{~A}$ \\
\hline \multicolumn{2}{|c|}{ Total mean } & & $20.51 \pm 0.84$ & $18.25 \pm 0.59$ & $2.59 \pm 0.12$ & $0.81 \pm 0.09$ & $7.88 \pm 0.27$ & $9.40 \pm 0.30$ & $325.97 \pm 7.71$ & $23.46 \pm 1.91$ & $41.91 \pm 1.64$ \\
\hline & Summer & & $18.94 \pm 1.52 \mathrm{~A}$ & $16.26 \pm 0.67 \mathrm{~A}$ & $2.66 \pm 0.23 \mathrm{AB}$ & $0.77 \pm 0.011 \mathrm{AB}$ & $7.23 \pm 0.39 \mathrm{~A}$ & $9.81 \pm 0.80 \mathrm{~A}$ & $346.38 \pm 16.07 \mathrm{~A}$ & $18.66 \pm 2.72 \mathrm{~A}$ & $49.07 \pm 3.72 \mathrm{~A}$ \\
\hline & Autumn & & $19.18 \pm 1.65 \mathrm{~A}$ & $18.11 \pm 0.97 \mathrm{~A}$ & $1.99 \pm 0.13 \mathrm{~B}$ & $0.58 \pm 0.06 \mathrm{~B}$ & $8.32 \pm 0.65 \mathrm{~A}$ & $8.71 \pm 0.66 \mathrm{~A}$ & $346.36 \pm 11.72 \mathrm{~A}$ & $23.72 \pm 3.18 \mathrm{~A}$ & $41.67 \pm 3.54 \mathrm{~A}$ \\
\hline & Winter & & $24.39 \pm 1.90 \mathrm{~A}$ & $18.69 \pm 1.74 \mathrm{~A}$ & $3.06 \pm 0.28 \mathrm{~A}$ & $1.40 \pm 0.30 \mathrm{~A}$ & $7.97 \pm 0.21 \mathrm{~A}$ & $10.26 \pm 0.45 \mathrm{~A}$ & $336.07 \pm 9.77 \mathrm{~A}$ & $26.33 \pm 5.31 \mathrm{~A}$ & $39.11 \pm 1.65 \mathrm{~A}$ \\
\hline \multirow{4}{*}{\multicolumn{2}{|c|}{ Total mean }} & & $20.51 \pm 0.84$ & $18.25 \pm 0.59$ & $2.59 \pm 0.12$ & $0.81 \pm 0.09$ & $7.88 \pm 0.27$ & $9.40 \pm 0.30$ & $325.97 \pm 7.71$ & $23.46 \pm 1.91$ & $41.91 \pm 1.64$ \\
\hline & & Mountain & $18.41 \pm 1.31 \mathrm{~B}$ & $16.71 \pm 0.98 \mathrm{~B}$ & $2.45 \pm 0.20 \mathrm{~A}$ & $0.57 \pm 0.07 \mathrm{~B}$ & $7.27 \pm 0.40 \mathrm{~B}$ & $8.90 \pm 0.48 \mathrm{~A}$ & $340.67 \pm 9.26 \mathrm{~A}$ & $23.63 \pm 2.71 \mathrm{~A}$ & $43.42 \pm 2.57 \mathrm{~A}$ \\
\hline & & Plain & $22.62 \pm 0.93 \mathrm{~A}$ & $19.79 \pm 0.57 \mathrm{~A}$ & $2.73 \pm 0.14 \mathrm{~A}$ & $1.04 \pm 0.16 \mathrm{~A}$ & $8.50 \pm 0.34 \mathrm{~A}$ & $9.91 \pm 0.35 \mathrm{~A}$ & $311.26 \pm 11.96 \mathrm{~B}$ & $23.29 \pm 2.73 \mathrm{~A}$ & $40.41 \pm 2.05 \mathrm{~A}$ \\
\hline & & Total mean & $20.51 \pm 0.84$ & $18.25 \pm 0.59$ & $2.59 \pm 0.12$ & $0.81 \pm 0.09$ & $7.88 \pm 0.27$ & $9.40 \pm 0.30$ & $325.97 \pm 7.71$ & $23.46 \pm 1.91$ & $41.91 \pm 1.64$ \\
\hline \multirow[t]{7}{*}{ Sage } & Summer & Mountain & $9.55 \pm 0.011^{Y}$ & $16.13 \pm 0.04$ fghij & $4.24 \pm 0.01 \mathrm{a}$ & $1.59 \pm 0.01 \mathrm{~b}$ & $4.65 \pm 0.18 \mathrm{ij}$ & $8.62 \pm 0.27$ defgh & $368.52 \pm 11.62 \mathrm{bcd}$ & $37.68 \pm 1.00 \mathrm{bcd}$ & $38.90 \pm 1.13 \mathrm{bcd}$ \\
\hline & & Plain & $16.12 \pm 0.24 \mathrm{hi}$ & $14.20 \pm 0.24 \mathrm{ijkl}$ & $1.44 \pm 0.03 \mathrm{fg}$ & $1.09 \pm 0.00 \mathrm{~d}$ & $7.76 \pm 0.38$ efgh & $7.02 \pm 0.38 \mathrm{ghi}$ & $301.86 \pm 8.22$ defg & $26.40 \pm 3.92$ def & $32.53 \pm 0.56 \mathrm{~cd}$ \\
\hline & Autumn & Mountain & $11.31 \pm 0.01 \mathrm{k}$ & $15.54 \pm 0.11$ hijk & $0.86 \pm 0.00 \mathrm{~h}$ & $0.25 \pm 0.02 \mathrm{jk}$ & $3.22 \pm 0.41 \mathrm{j}$ & $7.05 \pm 0.21 \mathrm{ghi}$ & $294.66 \pm 9.76$ defg & $35.81 \pm 1.13$ cde & $51.88 \pm 0.45 \mathrm{abc}$ \\
\hline & & Plain & $17.01 \pm 0.11 \mathrm{gh}$ & $19.71 \pm 0.21 \mathrm{def}$ & $2.22 \pm 0.08 \mathrm{de}$ & $0.92 \pm 0.04 \mathrm{e}$ & $11.29 \pm 0.31 \mathrm{ab}$ & $7.61 \pm 0.05$ fghi & $314.36 \pm 10.00 \mathrm{cdef}$ & $43.82 \pm 4.06 \mathrm{bc}$ & $36.42 \pm 3.90 \mathrm{bcd}$ \\
\hline & & Plain & $23.23 \pm 0.02 \mathrm{ef}$ & $15.58 \pm 0.26$ hij & $3.87 \pm 0.02 \mathrm{abc}$ & $0.83 \pm 0.00 \mathrm{e}$ & $8.88 \pm 0.96$ cdefgh & $9.57 \pm 0.19$ cdef & $347.60 \pm 10.88$ bcde & $61.76 \pm 4.52 \mathrm{a}$ & $34.19 \pm 1.51 \mathrm{~cd}$ \\
\hline & Spring & Mountain & $10.73 \pm 0.13 \mathrm{kl}$ & $11.99 \pm 0.11 \mathrm{kl}$ & $0.88 \pm 0.01 \mathrm{gh}$ & $0.46 \pm 0.00 \mathrm{gh}$ & $12.46 \pm 0.81 \mathrm{a}$ & $9.01 \pm 0.05$ cdefg & $412.43 \pm 12.79 \mathrm{ab}$ & $52.11 \pm 4.39 \mathrm{ab}$ & $52.38 \pm 7.33 \mathrm{abc}$ \\
\hline & & Plain & $24.26 \pm 0.06 \mathrm{de}$ & $21.85 \pm 0.25 \mathrm{bcd}$ & $3.42 \pm 0.12 \mathrm{c}$ & $0.36 \pm 0.02$ hij & $10.58 \pm 0.21 \mathrm{abc}$ & $11.07 \pm 0.13 b c$ & $260.80 \pm 3.58 \mathrm{fgh}$ & $37.17 \pm 0.20 \mathrm{bcd}$ & $43.62 \pm 3.84 \mathrm{abcd}$ \\
\hline \multirow[t]{8}{*}{ Sideritis } & Summer & Mountain & $17.96 \pm 0.34 \mathrm{~g}$ & $12.79 \pm 0.39 \mathrm{jkl}$ & $2.01 \pm 0.06$ ef & $0.27 \pm 0.00 \mathrm{jk}$ & $6.60 \pm 0.23 \mathrm{hi}$ & $9.31 \pm 1.16 \mathrm{cdef}$ & $356.51 \pm 34.04 \mathrm{bcd}$ & $14.59 \pm 3.07 \mathrm{fgh}$ & $47.61 \pm 5.11 \mathrm{abcd}$ \\
\hline & & Plain & $16.17 \pm 0.04 \mathrm{hi}$ & $19.29 \pm 2.93$ defg & $2.36 \pm 0.34 \mathrm{de}$ & $0.32 \pm 0.00 \mathrm{ijk}$ & $9.65 \pm 0.83$ bcde & $6.21 \pm 1.23 \mathrm{ij}$ & $466.64 \pm 4.26 \mathrm{a}$ & $15.65 \pm 3.42 \mathrm{fgh}$ & $49.39 \pm 6.45 \mathrm{abcd}$ \\
\hline & Autumn & Mountain & $15.11 \pm 0.76 \mathrm{i}$ & $17.08 \pm 0.27 \mathrm{efghi}$ & $2.17 \pm 0.12 \mathrm{e}$ & $0.24 \pm 0.02 \mathrm{jk}$ & $8.54 \pm 0.23$ cdefgh & $4.08 \pm 0.11 \mathrm{j}$ & $407.20 \pm 15.15 \mathrm{ab}$ & $6.25 \pm 1.22 \mathrm{~h}$ & $27.31 \pm 2.53 \mathrm{~cd}$ \\
\hline & & Plain & $16.37 \pm 0.04 \mathrm{hi}$ & $24.40 \pm 0.42 \mathrm{a}$ & $1.92 \pm 0.08$ ef & $0.61 \pm 0.06 \mathrm{f}$ & $10.51 \pm 0.69 \mathrm{abcd}$ & $10.84 \pm 0.25 \mathrm{bc}$ & $398.22 \pm 18.55 \mathrm{ab}$ & $13.24 \pm 0.82 \mathrm{fgh}$ & $64.44 \pm 4.89 \mathrm{ab}$ \\
\hline & Winter & Mountain & $18.17 \pm 0.05 \mathrm{~g}$ & $15.80 \pm 0.39$ ghij & $2.05 \pm 0.06 \mathrm{e}$ & $0.20 \pm 0.00 \mathrm{k}$ & $7.67 \pm 0.07$ efgh & $6.79 \pm 0.01 \mathrm{hi}$ & $364.98 \pm 3.34 \mathrm{bcd}$ & $7.39 \pm 0.30 \mathrm{gh}$ & $37.36 \pm 3.45 \mathrm{bcd}$ \\
\hline & & Plain & $23.95 \pm 0.02 \mathrm{ef}$ & $18.79 \pm 0.31$ defgh & $2.79 \pm 0.08 \mathrm{~d}$ & $1.48 \pm 0.04 \mathrm{bc}$ & $7.87 \pm 0.38$ efgh & $12.15 \pm 0.04 \mathrm{~b}$ & $276.22 \pm 13.39 \mathrm{efg}$ & $11.64 \pm 3.29 \mathrm{fgh}$ & $34.89 \pm 3.42 \mathrm{bcd}$ \\
\hline & Spring & Mountain & $17.14 \pm 0.17 \mathrm{gh}$ & $23.48 \pm 0.39 \mathrm{bc}$ & $3.90 \pm 0.04 \mathrm{abc}$ & $0.20 \pm 0.01 \mathrm{k}$ & $4.29 \pm 0.06 \mathrm{j}$ & $6.09 \pm 0.05 \mathrm{ij}$ & $297.69 \pm 2.85 \mathrm{defg}$ & $19.31 \pm 2.96 \mathrm{fgh}$ & $48.19 \pm 9.29 \mathrm{abcd}$ \\
\hline & & Plain & $18.09 \pm 0.17 \mathrm{~g}$ & $17.61 \pm 0.04$ efghi & $2.30 \pm 0.07 \mathrm{de}$ & $0.34 \pm 0.00 \mathrm{hij}$ & $3.86 \pm 0.10 \mathrm{j}$ & $9.11 \pm 0.38$ cdefg & $194.84 \pm 13.84 \mathrm{~h}$ & $14.01 \pm 0.09 \mathrm{fgh}$ & $30.44 \pm 0.31 \mathrm{~cd}$ \\
\hline
\end{tabular}


Table 4. Cont.

\begin{tabular}{|c|c|c|c|c|c|c|c|c|c|c|c|}
\hline Species & Season & Altitude & $\mathbf{N}$ & $\mathrm{K}$ & $\mathbf{P}$ & $\mathrm{Na}$ & $\mathrm{Ca}$ & $\mathrm{Mg}$ & $\mathrm{Fe}$ & $\mathrm{Zn}$ & $\mathrm{Cu}$ \\
\hline \multirow[t]{7}{*}{ Spearmint } & Summer & Mountain & $28.33 \pm 0.16 \mathrm{c}$ & $17.29 \pm 0.25$ efghi & $3.60 \pm 0.12 \mathrm{bc}$ & $0.46 \pm 0.01 \mathrm{gh}$ & $7.92 \pm 0.32 \mathrm{efgh}$ & $15.28 \pm 0.25 \mathrm{a}$ & $273.26 \pm 0.28$ efg & $5.53 \pm 0.34 \mathrm{~h}$ & $72.74 \pm 12.05 \mathrm{a}$ \\
\hline & & Plain & $25.52 \pm 0.05 \mathrm{~d}$ & $17.90 \pm 0.28$ efgh & $2.32 \pm 0.11 \mathrm{de}$ & $0.89 \pm 0.01 \mathrm{e}$ & $6.83 \pm 0.01 \mathrm{ghi}$ & $12.43 \pm 0.23 \mathrm{~b}$ & $311.51 \pm 0.58$ cdef & $12.11 \pm 2.96 \mathrm{fgh}$ & $53.26 \pm 3.63 \mathrm{abc}$ \\
\hline & Autumn & Mountain & $22.92 \pm 0.24 \mathrm{f}$ & $11.62 \pm 0.10 \mathrm{lkl}$ & $2.49 \pm 0.14 \mathrm{de}$ & $0.88 \pm 0.00 \mathrm{e}$ & $9.07 \pm 0.06$ bcdefg & $10.48 \pm 0.19 \mathrm{bcd}$ & $356.28 \pm 19.78 \mathrm{bcd}$ & $22.88 \pm 2.19$ defg & $33.21 \pm 10.41 \mathrm{~cd}$ \\
\hline & Winter & Mountain & $37.95 \pm 0.50 \mathrm{a}$ & $30.72 \pm 0.03 \mathrm{a}$ & $4.13 \pm 0.08 \mathrm{ab}$ & $1.43 \pm 0.02 c$ & $8.19 \pm 0.11$ efgh & $12.34 \pm 0.15 b$ & $321.87 \pm 23.57 \mathrm{cdef}$ & $23.92 \pm 3.94$ def & $39.33 \pm 6.98 \mathrm{bcd}$ \\
\hline & & Plain & $29.55 \pm 0.31 \mathrm{c}$ & $23.63 \pm 0.27 b c$ & $4.39 \pm 0.01 \mathrm{a}$ & $4.02 \pm 0.06 \mathrm{a}$ & $8.27 \pm 0.41$ defgh & $10.97 \pm 0.05 \mathrm{bc}$ & $321.48 \pm 17.18 \mathrm{cdef}$ & $5.97 \pm 0.68 \mathrm{~h}$ & $46.96 \pm 0.45 \mathrm{abcd}$ \\
\hline & Spring & Mountain & $18.26 \pm 0.05 \mathrm{~g}$ & $20.51 \pm 0.43$ cde & $1.94 \pm 0.06 \mathrm{ef}$ & $0.44 \pm 0.00 \mathrm{hi}$ & $7.73 \pm 0.29$ efgh & $7.97 \pm 0.07$ efghi & $250.34 \pm 3.78 \mathrm{fgh}$ & $10.81 \pm 0.28 \mathrm{fgh}$ & $30.16 \pm 8.03 \mathrm{~cd}$ \\
\hline & & Plain & $28.77 \pm 0.08 \mathrm{c}$ & $24.22 \pm 0.04 \mathrm{a}$ & $3.41 \pm 0.02 \mathrm{c}$ & $1.09 \pm 0.02 \mathrm{~d}$ & $9.17 \pm 0.48$ bcdef & $9.70 \pm 0.32 \mathrm{cdef}$ & $234.19 \pm 21.86 \mathrm{gh}$ & $17.37 \pm 0.03 \mathrm{fgh}$ & $22.05 \pm 3.87 \mathrm{~d}$ \\
\hline
\end{tabular}

Y values (means $\pm \mathrm{SE}, n=4$ ) in columns corresponding to the main factors (Altitude, Seasons and Species) followed by the
(Altitude, Seasons and Species), which are followed by the same lowercase letter, are not significantly different, $p<0.05$. 
Indeed, altitude (mountainous versus plain) did not affect the EO yield, which was averaged at $1.38 \pm 0.14 \%$. However, in comparison to plants harvested at higher elevations, Formisano et al. [56] found that chamomile harvested at low altitudes (81-89 m) yielded more EOs in comparison to plants harvested at higher elevations (i.e., 640-675 m). Norani et al. [32] showed that the lowest EO yield in Tussilago farfara (L.) was found at low altitudes (i.e., $229 \mathrm{~m}$ ), exhibiting at the same time the highest antioxidant activity when compared to plants growing at higher altitudes. It has been reported that as the altitude increases, the EO yield of Tanacetum polycephalum was found increased [57], but opposed findings were observed in case of Artemisia absinthium [58]. Mahomoodally et al. [59] found that EO yield varies throughout the year and was found lower in areas with less solar radiation. Different plant species and environmental conditions may provide diverse results. In the present study, altitude did not affect the EO yield and the antioxidant capacity throughout a calendar year, which is of great importance for farmers, if the species and the season period will not be taken into account.

Among the three examined species, when comparing the EO yield, regardless of the altitude and the collection season, sage had the highest yield $(2.56 \pm 0.23 \%)$, followed by spearmint $(1.25 \pm 0.16 \%)$ and then by sideritis $(0.34 \pm 0.04 \%)$. When considering the combined effect of the tested factors, plain sage in autumn had the highest $(4.76 \pm 0.19 \%)$ and plain spearmint in winter the lowest $(0.07 \pm 0.00 \%)$ EO yield, indicating the significance of the different species on this parameter, apart from the growing location. Specifically, the high altitude of the mountainous area decreased sage EOs yield in all seasons and spearmint EOs yield during summer-autumn (Table 3).

The effect of season and/or altitude on the EOs chemical composition of the examined MAP species is given in Tables 5-7. Sabbahi et al. [60] reported that the major components profile of the EOs was impacted by the altitude gradient and the variation of the EO composition was mostly related to the genetic variables. In the case of sage, EOs analysis revealed the presence of 32 individual compounds, representing a total percentage of $\geq 99.94 \%$ of the oil profile for the mountainous and plain plants. The most abundant class (ranged from $61.79 \%$ to $83.75 \%$ ) was oxygenated monoterpenes, followed by monoterpenes hydrocarbon (ranged from $14.33 \%$ to $28.34 \%$ ), oxygenated sesquiterpenes (ranged from $1.96 \%$ to $7.59 \%$ ), and sesquiterpenes hydrocarbons (ranged from $0.19 \%$ to $5.53 \%$ ) for the mountainous and plain plants throughout the seasons (Table 5). The major constituents of the examined sage EOs in decreasing order were 1,8-Cineole (17.48-29.20\%), Camphor (11.50-40.35\%), $\alpha$ Thujone (0.09-31.94\%), Camphene (2.98-8.82\%), $\beta$ Thujone $(0.02-7.51 \%)$, Limonene (0.95-7.18\%), Viridiflorol (1.67-6.95\%) and $\alpha$ Pinene (2.89-4.14\%). $\beta$ Pinene, $\beta$ Myrcene, $\beta$ Caryophyllene, Borneol, iso-Bornyl acetate and $\alpha$ Humulene varied between $1-4 \%$, while the rest of the compounds were identified in amounts lower than $1 \%$ of the total volatile components content (Table 5). Sage plants grown in the mountain had significantly higher content of 1,8-Cineole during summer but the highest content in plain areas occurred during spring season. Camphor content was greater in summer for mountainous and for plain (including autumn) sage while $\alpha$ Thujone was lower in summer and dominated during the rest of the year (Table 5). Holopainen et al. [61] have suggested that monoterpenes are highly volatile terpenoids emitted with warmer temperature than sesquiterpenes, as both 1,8-Cineole and Camphor belong to the monoterpenes class. The increased levels of 1,8-Cineole are of great importance, as it exhibits insecticidal, antimicrobial, antiallergic and anti-inflammatory, hepatoprotective, antitumoral and gastroprotective action, as has been reviewed by Caldas et al. [62]. In Lippia gracilis EOs, the percentages of Myrcene, 1,8-Cineole, and $\gamma$ Terpinene did not vary much between seasons, with the dry season revealing the highest percentage, with the exception of Myrcene, which had higher percentages in the rainy season [53]. In S. officinallis, the level of 1,8-Cineole and Camphor were constant until August, and then were slightly decreased [12], and this trend was also observed in the present work. According to Bedini et al. [63], $\alpha$ Thujone, Camphor and 1,8-Cineole were the main components of sage EOs, as they were identified in the present study. Cvetkovikj et al. [64] discovered four unique chemotypes in sage populations from 
Balkan countries, based on cis- $(\beta)$ and trans- $(\alpha)$ Thujone and Camphor content and suggested a significant correlation between EO composition and geographic characteristics. Not only environmental conditions, but cultivation practices as well (biofertilizers and biostimulants) can affect the EOs' composition [65]. Indeed, Thujones are toxic components and their absence from the EO of Thujone-containing species makes it intriguing to be investigated [66]. In this study, the lowest Thujone levels were found during the summer period in both mountainous and plain areas. Mineral application, including N, through compost increased the production of EO in basil plants, increased Linalool and Borneol content but decreased 1,8-Cineole levels [67]; a similar situation was observed in the sage plants of this study, grown in plain areas during autumn (i.e., high EO yield and low 1,8-Cineole levels).

In sideritis, EOs analysis revealed the presence of 35 individual compounds for both plain and mountainous plants, representing $\geq 99.95 \%$ of the total oil profile (Table 6). The main detected class was that of monoterpenes hydrocarbon (ranged from $58.63 \%$ to $93.23 \%$ ) followed by oxygenated sesquiterpenes (ranged from $5.30 \%$ to $39.77 \%$ ), while both sesquiterpenes hydrocarbons and oxygenated monoterpenes were in lower amounts ( $\leq 5.89 \%$ and $\leq 3.37 \%$, respectively). Accordingly, the major oil constituents in decreasing order were $\alpha$ Pinene (27.85-42.19\%), Valeranone (4.74-37.88\%), $\beta$ Phellandrene (12.40-33.69\%), $\beta$ Pinene (5.17-8.45\%) and Sabinene (1.66-4.60\%), whereas $\beta$ Caryophyllene, Caryophyllene9-epi, Cubenol-1-epi, $\beta$ Myrcene, 3-Carene and Terpinolene varied between 1-4\% (Table 6). Summer was the season that $\alpha$ Pinene content was decreased at both mountainous and plain areas, while $\beta$ Phellandrene content was increased in mountainous sideritis plants in summer. Botrel et al. [68] examined the seasonal effect on the chemical composition of Hyptis marrubioide EO and found that the highest levels of EO were found in summer, but the highest proportions of the major components were found in winter. It should be noted that the period with the maximum active compounds production may not be the same as the period with the highest biomass production. An active component is mainly determined by the degree of stress that the plants are exposed to, which triggers the production of EOs while lowering biomass, or vice versa [69].

Spearmint EOs analysis revealed the presence of 37 different constituents for the plain and mountainous plants throughout the four seasons, representing $\geq 99.94 \%$ of the total oil profile (Table 7). The most abundant class was oxygenated monoterpenes (ranged from $46.18 \%$ to $80.39 \%$ ), followed by monoterpenes hydrocarbon (ranged from $8.64 \%$ to $22.71 \%$ ), sesquiterpenes hydrocarbons (ranged from $2.09 \%$ to $9.95 \%$ ) and oxygenated sesquiterpenes $(\leq 0.285 \%)$ (Table 7$)$. The major constituents of the examined spearmint EO in decreasing order were Carvone (34.07-74.79\%), Dihydro carveol (0.10-16.25\%), Sabinene (0.58-15.54\%), D Limonene (4.09-14.60\%), cis Carvyl acetate (0.06-14.56\%) and 1,8-Cineole (3.79-8.84\%), whereas $\beta$ Pinene, neo Dihydro carveol, cis Carveol, $\beta$ Caryophyllene and Germacrene D varied between 1-4\% (Table 7). During winter time, Carvone was lower in mountainous area but greater in plain areas. In general, spearmint grown in plain areas revealed higher Carvone levels during summer, autumn and winter comparing to the relevant plants grown in mountainous areas. With less sunshine during winter, photosynthesis slows down, resulting in lower amounts of energy available for plant growth and development, resulting in lower production of metabolites as terpenes [68]. Instead, the energy provided by the plant is used to synthesize primary metabolites and maintain growth and development [69]. Several other studies found Carvone to be the most abundant spearmint EO component [70,71], but agronomic conditions, including salinity and water stress, may influence EOs yield and composition [44]. Carvone extracted from spearmint has a wide range of biocidal properties, including antioxidant, insecticidal, antifungal and antibacterial properties, as reviewed by Elmastaş et al. [70]. Talebi et al. [72] reported that the increased oxygenated compounds of EOs from Nepeta species from high altitudes ( $2290 \mathrm{~m}$ versus $1920 \mathrm{~m}$ ) can be related to the high doses of different UV radiation that lead to the development of oxidative stress in plants. Such trend could be the case for mountainous winter sage and sideritis, but not for spearmint. 
Table 5. Chemical composition (\%) of essential oils of sage plants.

\begin{tabular}{|c|c|c|c|c|c|c|c|c|c|}
\hline \multirow[b]{2}{*}{ Compound } & \multirow[b]{2}{*}{ RI } & \multicolumn{4}{|c|}{ Mountain } & \multicolumn{4}{|c|}{ Plain } \\
\hline & & Summer & Autumn & Winter & Spring & Summer & Autumn & Winter & Spring \\
\hline Tricyclene & 921 & $0.123 \pm 0.003 \mathrm{~b}$ & $0.186 \pm 0.005 \mathrm{a}$ & $0.192 \pm 0.005 \mathrm{a}$ & $0.071 \pm 0.006 \mathrm{~d}$ & $0.102 \pm 0.003 c$ & $0.101 \pm 0.003 c$ & $0.181 \pm 0.005 \mathrm{a}$ & $0.025 \pm 0.001 \mathrm{e}$ \\
\hline$\alpha$ Thujene & 926 & $0.139 \pm 0.004 \mathrm{c}$ & $0.128 \pm 0.003 c$ & $0.048 \pm 0.001 \mathrm{e}$ & $0.091 \pm 0.002 \mathrm{~d}$ & $0.079 \pm 0.002 \mathrm{~d}$ & $0.053 \pm 0.002 \mathrm{e}$ & $0.221 \pm 0.006 \mathrm{~b}$ & $0.289 \pm 0.002 \mathrm{a}$ \\
\hline$\alpha$ Pinene & 933 & $3.336 \pm 0.081 \mathrm{~cd}$ & $3.67 \pm 0.09 b c$ & $3.681 \pm 0.090 \mathrm{bc}$ & $2.892 \pm 0.009 \mathrm{~d}$ & $4.141 \pm 0.101 \mathrm{a}$ & $3.933 \pm 0.096 \mathrm{ab}$ & $4.088 \pm 0.100 \mathrm{ab}$ & $3.064 \pm 0.014 \mathrm{~d}$ \\
\hline Camphene & 948 & $6.562 \pm 0.16 \mathrm{c}$ & $5.911 \pm 0.145 c$ & $6.448 \pm 0.158 c$ & $4.812 \pm 0.007 \mathrm{~d}$ & $8.824 \pm 0.215 a$ & $7.58 \pm 0.185 b$ & $5.757 \pm 0.141 \mathrm{c}$ & $2.989 \pm 0.015 \mathrm{e}$ \\
\hline Sabinene & 973 & $0.039 \pm 0.001 \mathrm{~b}$ & $0.042 \pm 0.001 \mathrm{~b}$ & $0 \pm 0 \mathrm{~d}$ & $0.018 \pm 0.004 \mathrm{c}$ & $0 \pm 0 \mathrm{~d}$ & $0 \pm 0 \mathrm{~d}$ & $0.034 \pm 0.001 \mathrm{~b}$ & $0.056 \pm 0.001 \mathrm{a}$ \\
\hline$\beta$ Pinene & 977 & $2.364 \pm 0.058 c$ & $2.205 \pm 0.054 \mathrm{c}$ & $1.533 \pm 0.038$ ef & $1.857 \pm 0.003 \mathrm{~d}$ & $1.752 \pm 0.043 \mathrm{de}$ & $1.433 \pm 0.035 \mathrm{f}$ & $2.720 \pm 0.067 b$ & $3.380 \pm 0.007 a$ \\
\hline$\beta$ Myrcene & 989 & $1.452 \pm 0.036 \mathrm{f}$ & $1.645 \pm 0.040$ ef & $1.147 \pm 0.028 \mathrm{~g}$ & $1.958 \pm 0.006 \mathrm{~cd}$ & $2.183 \pm 0.053 c$ & $1.858 \pm 0.045 \mathrm{de}$ & $3.167 \pm 0.077 b$ & $3.791 \pm 0.024 \mathrm{a}$ \\
\hline$\alpha$ Phellandrene & 1004 & $0.052 \pm 0.001 \mathrm{c}$ & $0.020 \pm 0.001 \mathrm{~d}$ & $0.008 \pm 0.000 \mathrm{e}$ & $0 \pm 0 \mathrm{e}$ & $0.194 \pm 0.005 \mathrm{a}$ & $0.105 \pm 0.003 \mathrm{~b}$ & $0.058 \pm 0.002 \mathrm{c}$ & $0.023 \pm 0.000 \mathrm{~d}$ \\
\hline$\alpha$ Terpinene & 1017 & $0.164 \pm 0.004 \mathrm{c}$ & $0.103 \pm 0.003 \mathrm{~d}$ & $0.038 \pm 0.001 \mathrm{e}$ & $0.110 \pm 0.009 \mathrm{~d}$ & $0.154 \pm 0.004 \mathrm{c}$ & $0.159 \pm 0.004 \mathrm{c}$ & $0.394 \pm 0.01 \mathrm{~b}$ & $0.433 \pm 0.003 \mathrm{a}$ \\
\hline p Cymene & 1024 & $0.428 \pm 0.011 \mathrm{e}$ & $1.482 \pm 0.036 \mathrm{~b}$ & $1.662 \pm 0.041 \mathrm{a}$ & $1.095 \pm 0.011 \mathrm{c}$ & $0.243 \pm 0.006 \mathrm{f}$ & $0.763 \pm 0.019 \mathrm{~d}$ & $1.404 \pm 0.035 \mathrm{~b}$ & $0.402 \pm 0.004 \mathrm{e}$ \\
\hline Limonene & 1028 & $3.215 \pm 0.078 \mathrm{c}$ & $1.446 \pm 0.035 \mathrm{~d}$ & $1.407 \pm 0.034 \mathrm{de}$ & $1.167 \pm 0.022 \mathrm{de}$ & $7.187 \pm 0.176 a$ & $5.420 \pm 0.132 b$ & $1.568 \pm 0.038 \mathrm{~d}$ & $0.955 \pm 0.014 \mathrm{e}$ \\
\hline 1,8-Cineole & 1031 & $29.206 \pm 0.713 a$ & $21.605 \pm 0.527 b$ & $21.39 \pm 0.522 b$ & $21.329 \pm 0.129 b$ & $19.737 \pm 0.482 b c$ & $17.482 \pm 0.426 \mathrm{c}$ & $21.163 \pm 0.517 b$ & $27.762 \pm 0.005 a$ \\
\hline$\gamma$ Terpinene & 1058 & $0.278 \pm 0.007 \mathrm{c}$ & $0.146 \pm 0.004 \mathrm{e}$ & $0.058 \pm 0.002 \mathrm{f}$ & $0.257 \pm 0.008 \mathrm{~cd}$ & $0.257 \pm 0.006 \mathrm{~cd}$ & $0.222 \pm 0.006 \mathrm{~d}$ & $0.604 \pm 0.015 \mathrm{~b}$ & $0.858 \pm 0.000 \mathrm{a}$ \\
\hline Linalool & 1100 & $0.075 \pm 0.002 \mathrm{c}$ & $0.074 \pm 0.002 \mathrm{c}$ & $0 \pm 0 \mathrm{e}$ & $0.029 \pm 0.003 \mathrm{~d}$ & $0.253 \pm 0.006 \mathrm{a}$ & $0.161 \pm 0.004 \mathrm{~b}$ & $0.035 \pm 0.001 \mathrm{~d}$ & $0.065 \pm 0.001 \mathrm{c}$ \\
\hline$\alpha$ Thujone & 1105 & $0.274 \pm 0.007 \mathrm{~d}$ & $31.286 \pm 0.763 \mathrm{a}$ & $31.948 \pm 0.779 a$ & $29.678 \pm 0.183 \mathrm{a}$ & $0.093 \pm 0.003 \mathrm{~d}$ & $11.079 \pm 0.27 c$ & $29.977 \pm 0.731 \mathrm{a}$ & $23.103 \pm 0.061 \mathrm{~b}$ \\
\hline$\beta$ Thujone & 1122 & $0.023 \pm 0.001 \mathrm{e}$ & $5.802 \pm 0.142 c$ & $5.41 \pm 0.132 c$ & $5.503 \pm 0.009 c$ & $0.045 \pm 0.001 \mathrm{e}$ & $2.866 \pm 0.07 \mathrm{~d}$ & $6.623 \pm 0.162 b$ & $7.506 \pm 0.007 a$ \\
\hline trans Sabinol & 1138 & $0 \pm 0 \mathrm{e}$ & $0.158 \pm 0.004 \mathrm{a}$ & $0.017 \pm 0.001 \mathrm{de}$ & $0.027 \pm 0.007 \mathrm{~d}$ & $0 \pm 0 \mathrm{e}$ & $0.078 \pm 0.002 \mathrm{bc}$ & $0.087 \pm 0.002 \mathrm{~b}$ & $0.064 \pm 0.002 c$ \\
\hline Camphor & 1145 & $40.358 \pm 0.984 a$ & $19.794 \pm 0.483 b c$ & $23.484 \pm 0.573 b$ & $18.963 \pm 0.118 \mathrm{c}$ & $39.889 \pm 0.973 \mathrm{a}$ & $40.055 \pm 0.977 a$ & $13.959 \pm 0.341 \mathrm{~d}$ & $11.502 \pm 0.043 \mathrm{~d}$ \\
\hline Borneol & 1166 & $0.568 \pm 0.014 \mathrm{e}$ & $1.744 \pm 0.043 \mathrm{ab}$ & $1.037 \pm 0.026 \mathrm{c}$ & $1.807 \pm 0.041 \mathrm{a}$ & $0.37 \pm 0.009 \mathrm{f}$ & $0.747 \pm 0.019 \mathrm{~d}$ & $1.595 \pm 0.039 c$ & $0.601 \pm 0.01 \mathrm{de}$ \\
\hline Terpinen-4-ol & 1178 & $0.545 \pm 0.014 \mathrm{c}$ & $0.715 \pm 0.018 \mathrm{a}$ & $0.418 \pm 0.01 \mathrm{~d}$ & $0.356 \pm 0.022 \mathrm{~d}$ & $0.584 \pm 0.015 \mathrm{bc}$ & $0.654 \pm 0.016 \mathrm{ab}$ & $0.574 \pm 0.014 \mathrm{bc}$ & $0.2 \pm 0.001 \mathrm{e}$ \\
\hline p-Cymen-8-ol & 1185 & $0.099 \pm 0.003 \mathrm{c}$ & $0.032 \pm 0.001 \mathrm{~d}$ & $0.011 \pm 0.001 \mathrm{e}$ & $0 \pm 0 \mathrm{e}$ & $0.250 \pm 0.006 \mathrm{~b}$ & $0.28 \pm 0.007 a$ & $0 \pm 0 \mathrm{e}$ & $0 \pm 0 \mathrm{e}$ \\
\hline$\alpha$ Terpineol & 1191 & $0.098 \pm 0.003 c$ & $0.074 \pm 0.002 \mathrm{~cd}$ & $0.019 \pm 0.001 \mathrm{e}$ & $0.012 \pm 0.012 \mathrm{e}$ & $0.271 \pm 0.007 \mathrm{a}$ & $0.178 \pm 0.005 b$ & $0.025 \pm 0.001 \mathrm{e}$ & $0.065 \pm 0.001 \mathrm{~d}$ \\
\hline iso-Bornyl acetate & 1284 & $0.793 \pm 0.02 b$ & $0.326 \pm 0.008 \mathrm{~d}$ & $0.168 \pm 0.005 \mathrm{e}$ & $0.077 \pm 0.008 \mathrm{e}$ & $2.669 \pm 0.065 a$ & $0.888 \pm 0.022 \mathrm{~b}$ & $0.147 \pm 0.004 \mathrm{e}$ & $0.477 \pm 0.001 \mathrm{c}$ \\
\hline trans Sabinyl acetate & 1293 & $0 \pm 0 \mathrm{~d}$ & $0.051 \pm 0.001 \mathrm{c}$ & $0.028 \pm 0.001 \mathrm{c}$ & $0 \pm 0 \mathrm{~d}$ & $0.058 \pm 0.002 \mathrm{~b}$ & $0.19 \pm 0.005 \mathrm{a}$ & $0.050 \pm 0.002 \mathrm{~b}$ & $0.029 \pm 0.002 c$ \\
\hline$\alpha$ Terpinyl acetate & 1349 & $0.196 \pm 0.005 \mathrm{c}$ & $0.215 \pm 0.006 \mathrm{c}$ & $0 \pm 0 \mathrm{~d}$ & $0 \pm 0 \mathrm{~d}$ & $0.573 \pm 0.014 \mathrm{a}$ & $0.266 \pm 0.007 \mathrm{~b}$ & $0 \pm 0 \mathrm{~d}$ & $0 \pm 0 \mathrm{~d}$ \\
\hline$\beta$ Caryophyllene & 1425 & $2.764 \pm 0.068 b$ & $0 \pm 0 \mathrm{e}$ & $0.032 \pm 0.001 \mathrm{e}$ & $1.371 \pm 0.047 \mathrm{c}$ & $2.741 \pm 0.067 \mathrm{~b}$ & $0.95 \pm 0.024 \mathrm{~d}$ & $0.813 \pm 0.02 \mathrm{~d}$ & $3.105 \pm 0.017 a$ \\
\hline
\end{tabular}


Table 5. Cont.

\begin{tabular}{|c|c|c|c|c|c|c|c|c|c|}
\hline \multirow[b]{2}{*}{ Compound } & \multirow[b]{2}{*}{ RI } & \multicolumn{4}{|c|}{ Mountain } & \multicolumn{4}{|c|}{ Plain } \\
\hline & & Summer & Autumn & Winter & Spring & Summer & Autumn & Winter & Spring \\
\hline$\delta$ Cadinene & 1522 & $0.039 \pm 0.001 \mathrm{c}$ & $0 \pm 0 \mathrm{e}$ & $0 \pm 0 \mathrm{e}$ & $0 \pm 0 \mathrm{e}$ & $0.091 \pm 0.002 \mathrm{a}$ & $0.057 \pm 0.002 \mathrm{~b}$ & $0.024 \pm 0.001 \mathrm{~d}$ & $0 \pm 0 \mathrm{e}$ \\
\hline Caryophyllene oxide & 1587 & $0.519 \pm 0.013 \mathrm{a}$ & $0.234 \pm 0.006 \mathrm{c}$ & $0.029 \pm 0.001 \mathrm{de}$ & $0.043 \pm 0.016 \mathrm{de}$ & $0.458 \pm 0.0110 \mathrm{~b}$ & $0.227 \pm 0.006 c$ & $0.073 \pm 0.002 \mathrm{~d}$ & $0 \pm 0 \mathrm{e}$ \\
\hline Viridiflorol & 1591 & $6.953 \pm 0.170 \mathrm{a}$ & $2.189 \pm 0.054 c$ & $1.676 \pm 0.041 \mathrm{c}$ & $4.919 \pm 0.129 b$ & $4.476 \pm 0.110 \mathrm{~b}$ & $2.137 \pm 0.053 \mathrm{c}$ & $4.463 \pm 0.109 \mathrm{~b}$ & $6.515 \pm 0.017 \mathrm{a}$ \\
\hline Humulene epoxide II & 1608 & $0.121 \pm 0.003 c$ & $0.499 \pm 0.012 \mathrm{a}$ & $0.261 \pm 0.007 \mathrm{~b}$ & $0.234 \pm 0.033 b$ & $0.114 \pm 0.003 c$ & $0.214 \pm 0.005 \mathrm{~b}$ & $0.515 \pm 0.013 \mathrm{a}$ & $0.007 \pm 0.007 \mathrm{~d}$ \\
\hline \multicolumn{2}{|l|}{ Total Identified } & $99.850 \pm 0.02$ & $99.711 \pm 0.02$ & $99.881 \pm 0.02$ & $99.906 \pm 0.01$ & $99.947 \pm 0.02$ & $99.881 \pm 0.02$ & $99.757 \pm 0.02$ & $99.907 \pm 0.005$ \\
\hline \multicolumn{2}{|c|}{ Monoterpenes hydrocarbons } & $18.904 \pm 0.461 \mathrm{~cd}$ & $17.025 \pm 0.416 \mathrm{de}$ & $16.25 \pm 0.396$ ef & $14.334 \pm 0.043 \mathrm{f}$ & $28.34 \pm 0.691 \mathrm{a}$ & $22.985 \pm 0.561 \mathrm{~b}$ & $20.279 \pm 0.495 c$ & $16.434 \pm 0.089 \mathrm{def}$ \\
\hline \multicolumn{2}{|c|}{ Sesquiterpenes hydrocarbons } & $3.535 \pm 0.086 c$ & $0.308 \pm 0.008 \mathrm{f}$ & $0.196 \pm 0.005 \mathrm{f}$ & $2.586 \pm 0.085 \mathrm{~d}$ & $3.886 \pm 0.095 \mathrm{~b}$ & $1.764 \pm 0.043 \mathrm{e}$ & $2.642 \pm 0.065 \mathrm{~d}$ & $5.531 \pm 0.02 \mathrm{a}$ \\
\hline \multicolumn{2}{|c|}{ Oxygenated monoterpenes } & $71.305 \pm 1.739 c$ & $81.338 \pm 1.984 \mathrm{ab}$ & $83.75 \pm 2.043 a$ & $77.714 \pm 0.323 \mathrm{abc}$ & $61.793 \pm 1.507 \mathrm{~d}$ & $73.689 \pm 1.797 \mathrm{bc}$ & $74.064 \pm 1.807 \mathrm{bc}$ & $70.915 \pm 0.093 c$ \\
\hline \multicolumn{2}{|c|}{ Oxygenated sesquiterpenes } & $7.593 \pm 0.185 \mathrm{a}$ & $2.922 \pm 0.072 \mathrm{~d}$ & $1.966 \pm 0.048 \mathrm{e}$ & $5.196 \pm 0.178 c$ & $5.048 \pm 0.123 c$ & $2.577 \pm 0.063 \mathrm{de}$ & $5.051 \pm 0.124 \mathrm{c}$ & $6.522 \pm 0.024 b$ \\
\hline \multicolumn{2}{|l|}{ Others } & $0.988 \pm 0.024 \mathrm{c}$ & $0.592 \pm 0.015 \mathrm{~d}$ & $0.196 \pm 0.005 \mathrm{e}$ & $0.077 \pm 0.008 \mathrm{e}$ & $3.359 \pm 0.082 \mathrm{a}$ & $1.343 \pm 0.033 \mathrm{~b}$ & $0.196 \pm 0.005 \mathrm{e}$ & $0.507 \pm 0.003 \mathrm{~d}$ \\
\hline
\end{tabular}

Values $(n=3)$ in rows followed by the same letter are not significantly different, $p \leq 0.05$. In bold indicated EO components $>1 \%$.

Table 6. Chemical composition (\%) of essential oils of sideritis plants.

\begin{tabular}{|c|c|c|c|c|c|c|c|c|c|}
\hline \multirow[b]{2}{*}{ Compound } & \multirow[b]{2}{*}{ RI } & \multicolumn{4}{|c|}{ Mountain } & \multicolumn{4}{|c|}{ Plain } \\
\hline & & Summer & Autumn & Winter & Spring & Summer & Autumn & Winter & Spring \\
\hline$\alpha$ Thujene & 926 & $0.642 \pm 0.019 c$ & $0.807 \pm 0.024$ aа & $0.153 \pm 0.005 \mathrm{~cd}$ & $0.211 \pm 0.004 \mathrm{de}$ & $0.710 \pm 0.039 \mathrm{ab}$ & $0.701 \pm 0.021 \mathrm{ab}$ & $0.341 \pm 0.01 \mathrm{~cd}$ & $0.474 \pm 0.002 c$ \\
\hline$\alpha$ Pinene & 933 & $27.855 \pm 0.811 \mathrm{~d}$ & $37.753 \pm 1.100 \mathrm{~b}$ & $31.688 \pm 0.923 \mathrm{~cd}$ & $42.196 \pm 0.151 \mathrm{a}$ & $27.985 \pm 0.589 \mathrm{~d}$ & $35.378 \pm 1.031 \mathrm{bc}$ & $33.84 \pm 0.986 b c$ & $32.68 \pm 0.151 \mathrm{c}$ \\
\hline Camphene & 948 & $0.04 \pm 0.001 \mathrm{c}$ & $0.105 \pm 0.003 \mathrm{a}$ & $0 \pm 0 \mathrm{~d}$ & $0.023 \pm 0.006 c$ & $0.083 \pm 0.005 \mathrm{~b}$ & $0.071 \pm 0.002 b$ & $0 \pm 0 \mathrm{~d}$ & $0.040 \pm 0.001 \mathrm{c}$ \\
\hline Sabinene & 973 & $4.005 \pm 0.117 \mathrm{a}$ & $3.944 \pm 0.115 a$ & $1.663 \pm 0.049 c$ & $2.531 \pm 0.016 b$ & $4.602 \pm 0.207 a$ & $4.326 \pm 0.126 a$ & $2.610 \pm 0.076 b$ & $3.975 \pm 0.018 a$ \\
\hline$\beta$ Pinene & 977 & $5.176 \pm 0.151 d$ & $8.458 \pm 0.247 a$ & $5.24 \pm 0.153 \mathrm{~cd}$ & $7.291 \pm 0.038 b$ & $7.136 \pm 0.262 b$ & $7.173 \pm 0.209 b$ & $6.292 \pm 0.184 b c$ & $5.961 \pm 0.001 \mathrm{~cd}$ \\
\hline$\beta$ Myrcene & 989 & $2.711 \pm 0.079 b$ & $3.616 \pm 0.106 a$ & $0.789 \pm 0.023 \mathrm{~d}$ & $0.432 \pm 0.010 \mathrm{~d}$ & $2.459 \pm 0.12 b$ & $2.859 \pm 0.083 b$ & $0.477 \pm 0.014 \mathrm{~d}$ & $1.348 \pm 0.010 \mathrm{c}$ \\
\hline$\alpha$ Phellandrene & 1005 & $0.843 \pm 0.025 \mathrm{a}$ & $0.775 \pm 0.023 \mathrm{a}$ & $0.056 \pm 0.002 \mathrm{~d}$ & $0.038 \pm 0.008 \mathrm{~d}$ & $0.886 \pm 0.038 \mathrm{a}$ & $0.807 \pm 0.024 \mathrm{a}$ & $0.203 \pm 0.006 c$ & $0.427 \pm 0.009 \mathrm{~b}$ \\
\hline 3-Carene & 1013 & $2.982 \pm 0.087 \mathrm{ab}$ & $3.153 \pm 0.092 a$ & $1.036 \pm 0.030 \mathrm{~d}$ & $0.569 \pm 0.020 \mathrm{e}$ & $2.294 \pm 0.016 c$ & $2.775 \pm 0.081 b$ & $1.126 \pm 0.033 \mathrm{~d}$ & $2.148 \pm 0.002 c$ \\
\hline$\alpha$ Terpinene & 1017 & $0.217 \pm 0.007 \mathrm{a}$ & $0.172 \pm 0.005 b$ & $0 \pm 0 \mathrm{e}$ & $0 \pm 0 \mathrm{e}$ & $0.179 \pm 0.005 \mathrm{~b}$ & $0.158 \pm 0.005 b$ & $0.112 \pm 0.004 c$ & $0.071 \pm 0.000 \mathrm{~d}$ \\
\hline p Cymene & 1022 & $0.040 \pm 0.001 \mathrm{~cd}$ & $0.057 \pm 0.002 \mathrm{bc}$ & $0 \pm 0 \mathrm{~d}$ & $0.028 \pm 0.006 \mathrm{~cd}$ & $0.155 \pm 0.006 \mathrm{a}$ & $0.044 \pm 0.002 \mathrm{~cd}$ & $0.094 \pm 0.003 \mathrm{~b}$ & $0.022 \pm 0.022 \mathrm{~cd}$ \\
\hline$o$ Cymene & 1024 & $0.285 \pm 0.008 \mathrm{~d}$ & $0.494 \pm 0.014 b$ & $0.345 \pm 0.010 c$ & $0.250 \pm 0.001 \mathrm{~d}$ & $0 \pm 0 \mathrm{e}$ & $0.369 \pm 0.011 c$ & $0.634 \pm 0.019 \mathrm{a}$ & $0.235 \pm 0.002 \mathrm{~d}$ \\
\hline
\end{tabular}


Table 6. Cont.

\begin{tabular}{|c|c|c|c|c|c|c|c|c|c|}
\hline \multirow[b]{2}{*}{ Compound } & \multirow[b]{2}{*}{ RI } & \multicolumn{4}{|c|}{ Mountain } & \multicolumn{4}{|c|}{ Plain } \\
\hline & & Summer & Autumn & Winter & Spring & Summer & Autumn & Winter & Spring \\
\hline$\beta$ Phellandrene & 1029 & $33.696 \pm 0.982 \mathrm{a}$ & $30.551 \pm 0.890 \mathrm{ab}$ & $17.235 \pm 0.502 \mathrm{~d}$ & $12.400 \pm 0.054 \mathrm{e}$ & $26.659 \pm 0.644 \mathrm{c}$ & $30.900 \pm 0.900 \mathrm{ab}$ & $13.75 \pm 0.401 \mathrm{de}$ & $27.392 \pm 0.033 b c$ \\
\hline$\gamma$ Terpinene & 1058 & $0.495 \pm 0.015 \mathrm{a}$ & $0.465 \pm 0.014 \mathrm{a}$ & $0 \pm 0 \mathrm{~d}$ & $0.038 \pm 0.008 \mathrm{~d}$ & $0.376 \pm 0.013 \mathrm{~b}$ & $0.371 \pm 0.011 \mathrm{~b}$ & $0.261 \pm 0.008 \mathrm{c}$ & $0.246 \pm 0.003 \mathrm{c}$ \\
\hline Terpinolene & 1089 & $3.365 \pm 0.098 \mathrm{a}$ & $2.889 \pm 0.084 b$ & $0.431 \pm 0.013 \mathrm{f}$ & $0.129 \pm 0.010 \mathrm{~g}$ & $2.481 \pm 0.036 \mathrm{c}$ & $2.890 \pm 0.084 b$ & $0.854 \pm 0.025 \mathrm{e}$ & $1.535 \pm 0.005 \mathrm{~d}$ \\
\hline trans Sabinene hydrate & 1100 & $0.115 \pm 0.003 \mathrm{c}$ & $0.110 \pm 0.003 \mathrm{c}$ & $0.341 \pm 0.01 \mathrm{~b}$ & $0.008 \pm 0.008 \mathrm{~d}$ & $0.472 \pm 0.014 \mathrm{a}$ & $0.115 \pm 0.004 \mathrm{c}$ & $0.120 \pm 0.004 \mathrm{c}$ & $0.088 \pm 0.005 \mathrm{c}$ \\
\hline$\alpha$ Thujone & 1106 & $0 \pm 0 \mathrm{~b}$ & $0.739 \pm 0.022 \mathrm{a}$ & $0.77 \pm 0.023 \mathrm{a}$ & $0.057 \pm 0.015 \mathrm{~b}$ & $0 \pm 0 \mathrm{~b}$ & $0 \pm 0 \mathrm{~b}$ & $0 \pm 0 \mathrm{~b}$ & $0 \pm 0 \mathrm{~b}$ \\
\hline$\alpha$ Campholenal & 1127 & $0.042 \pm 0.001 \mathrm{f}$ & $0.069 \pm 0.002 \mathrm{ed}$ & $0.418 \pm 0.013 c$ & $0.773 \pm 0.023 \mathrm{~b}$ & $0 \pm 0 \mathrm{f}$ & $0.204 \pm 0.006 \mathrm{~d}$ & $1.413 \pm 0.041 \mathrm{a}$ & $0.141 \pm 0.002$ de \\
\hline Camphor & 1145 & $0.173 \pm 0.005 b$ & $0.185 \pm 0.006 b$ & $0 \pm 0 \mathrm{c}$ & $0.638 \pm 0.044 \mathrm{a}$ & $0 \pm 0 \mathrm{c}$ & $0.049 \pm 0.001 \mathrm{c}$ & $0 \pm 0 \mathrm{c}$ & $0.024 \pm 0.000 \mathrm{c}$ \\
\hline pinocarvone & 1163 & $0 \pm 0 \mathrm{~d}$ & $0 \pm 0 \mathrm{~d}$ & $0 \pm 0 \mathrm{~d}$ & $0.172 \pm 0.012 \mathrm{~b}$ & $0 \pm 0 \mathrm{~d}$ & $0.059 \pm 0.002 c$ & $0.429 \pm 0.013 \mathrm{a}$ & $0.013 \pm 0.001 \mathrm{~d}$ \\
\hline Terpinen-4-ol & 1178 & $0.069 \pm 0.002 \mathrm{~cd}$ & $0.345 \pm 0.011 \mathrm{a}$ & $0 \pm 0 \mathrm{e}$ & $0 \pm 0 \mathrm{e}$ & $0.225 \pm 0.018 \mathrm{~b}$ & $0.120 \pm 0.004 \mathrm{c}$ & $0.222 \pm 0.007 \mathrm{~b}$ & $0.034 \pm 0.002 \mathrm{de}$ \\
\hline Myrtenal & 1193 & $0 \pm 0 \mathrm{~d}$ & $0 \pm 0 \mathrm{~d}$ & $0.066 \pm 0.002 \mathrm{c}$ & $0.138 \pm 0.013 b$ & $0 \pm 0 \mathrm{~d}$ & $0.069 \pm 0.002 \mathrm{c}$ & $0.588 \pm 0.018 \mathrm{a}$ & $0.015 \pm 0.001 \mathrm{~d}$ \\
\hline Decanal & 1204 & $0.435 \pm 0.013 \mathrm{a}$ & $0.167 \pm 0.005 c$ & $0 \pm 0 \mathrm{~d}$ & $0 \pm 0 \mathrm{~d}$ & $0.174 \pm 0.002 \mathrm{c}$ & $0.224 \pm 0.007 \mathrm{~b}$ & $0 \pm 0 \mathrm{~d}$ & $0 \pm 0 \mathrm{~d}$ \\
\hline Carvone & 1244 & $0.806 \pm 0.024 \mathrm{a}$ & $0.176 \pm 0.005 \mathrm{~b}$ & $0.318 \pm 0.009 \mathrm{~b}$ & $0 \pm 0 \mathrm{~b}$ & $0.368 \pm 0.139 \mathrm{~b}$ & $0.090 \pm 0.003 \mathrm{~b}$ & $0.198 \pm 0.006 \mathrm{~b}$ & $0.024 \pm 0.001 \mathrm{~b}$ \\
\hline$\beta$ Bourbonene & 1386 & $0.169 \pm 0.005 c$ & $0.140 \pm 0.004 \mathrm{c}$ & $0 \pm 0 \mathrm{~d}$ & $0.229 \pm 0.006 \mathrm{~b}$ & $0.169 \pm 0.008 \mathrm{c}$ & $0.154 \pm 0.004 \mathrm{c}$ & $0.3 \pm 0.009 \mathrm{a}$ & $0.158 \pm 0.001 \mathrm{c}$ \\
\hline Caryophyllene-9-epi & 1479 & $1.350 \pm 0.039 a$ & $0.400 \pm 0.012 c$ & $0 \pm 0 \mathrm{~d}$ & $0 \pm 0 \mathrm{~d}$ & $0.974 \pm 0.071 \mathrm{~b}$ & $0.869 \pm 0.025 b$ & $0 \pm 0 \mathrm{~d}$ & $0 \pm 0 \mathrm{~d}$ \\
\hline Germacrene D & 1495 & $0.453 \pm 0.013 \mathrm{~d}$ & $0.223 \pm 0.007 \mathrm{~d}$ & $0.200 \pm 0.006 \mathrm{~d}$ & $1.276 \pm 0.023 \mathrm{~b}$ & $1.759 \pm 0.090 \mathrm{a}$ & $0.972 \pm 0.028 \mathrm{c}$ & $0.974 \pm 0.029 \mathrm{c}$ & $1.050 \pm 0.032 \mathrm{bc}$ \\
\hline Germacrene B & 1559 & $0.756 \pm 0.022 b c$ & $0.313 \pm 0.009 \mathrm{e}$ & $0.155 \pm 0.005 \mathrm{f}$ & $0.844 \pm 0.052 \mathrm{~b}$ & $0 \pm 0 \mathrm{~g}$ & $0.474 \pm 0.014 \mathrm{~d}$ & $1.542 \pm 0.045 \mathrm{a}$ & $0.671 \pm 0.019 c$ \\
\hline Caryophyllene oxide & 1587 & $0.467 \pm 0.014 \mathrm{~d}$ & $0 \pm 0 \mathrm{e}$ & $1.592 \pm 0.046 \mathrm{~b}$ & $1.590 \pm 0.029 b$ & $0.127 \pm 0.005 \mathrm{e}$ & $0.103 \pm 0.003 \mathrm{e}$ & $3.435 \pm 0.100 \mathrm{a}$ & $0.699 \pm 0.006 c$ \\
\hline Viridiflorol & 1592 & $0.242 \pm 0.007 \mathrm{~b}$ & $0 \pm 0 \mathrm{c}$ & $0 \pm 0 \mathrm{c}$ & $0 \pm 0 \mathrm{c}$ & $0.206 \pm 0.020 \mathrm{~b}$ & $0 \pm 0 \mathrm{c}$ & $0.309 \pm 0.009 \mathrm{a}$ & $0 \pm 0 \mathrm{c}$ \\
\hline Cubenol-1-epi & 1617 & $1.171 \pm 0.035 b$ & $0.557 \pm 0.016 \mathrm{~cd}$ & $0.297 \pm 0.009 \mathrm{~d}$ & $1.275 \pm 0.006 \mathrm{~b}$ & $1.834 \pm 0.113 \mathrm{a}$ & $0.756 \pm 0.022 \mathrm{c}$ & $1.456 \pm 0.043 \mathrm{~b}$ & $1.229 \pm 0.023 b$ \\
\hline Valeranone & 1673 & $10.393 \pm 0.303 \mathrm{de}$ & $4.745 \pm 0.138 \mathrm{f}$ & $37.884 \pm 1.104 \mathrm{a}$ & $21.853 \pm 0.579 b$ & $11.242 \pm 0.848 \mathrm{~d}$ & $6.972 \pm 0.203$ ef & $24.593 \pm 0.717 b$ & $14.953 \pm 0.050 \mathrm{c}$ \\
\hline$\delta$ Dodecalactone & 1704 & $0.222 \pm 0.007 \mathrm{~cd}$ & $0.158 \pm 0.005 \mathrm{~d}$ & $0.484 \pm 0.014 \mathrm{~b}$ & $0.913 \pm 0.059 \mathrm{a}$ & $0.407 \pm 0.068 \mathrm{bc}$ & $0.235 \pm 0.007 \mathrm{~cd}$ & $1.062 \pm 0.031 \mathrm{a}$ & $0.394 \pm 0.031 \mathrm{bcd}$ \\
\hline Isokaurene & 1990 & $0.465 \pm 0.014 \mathrm{a}$ & $0.317 \pm 0.009 \mathrm{~b}$ & $0 \pm 0 \mathrm{c}$ & $0 \pm 0 \mathrm{c}$ & $0.292 \pm 0.045 \mathrm{~b}$ & $0.505 \pm 0.015 \mathrm{a}$ & $0 \pm 0 \mathrm{c}$ & $0.011 \pm 0.011 \mathrm{c}$ \\
\hline Sclareol & 2135 & $0.666 \pm 0.02 \mathrm{a}$ & $0.103 \pm 0.003 \mathrm{~cd}$ & $0 \pm 0 \mathrm{~d}$ & $0.106 \pm 0.012 \mathrm{~cd}$ & $0.302 \pm 0.082 \mathrm{bc}$ & $0.502 \pm 0.015 \mathrm{ab}$ & $0 \pm 0 \mathrm{~d}$ & $0 \pm 0 \mathrm{~d}$ \\
\hline \multicolumn{2}{|l|}{ Total Identified } & $99.764 \pm 0.020$ & $99.951 \pm 0.020$ & $99.021 \pm 0.020$ & $99.003 \pm 0.028$ & $98.04 \pm 0.297$ & $99.863 \pm 0.0020$ & $97.529 \pm 0.020$ & $99.444 \pm 0.057$ \\
\hline \multicolumn{2}{|c|}{ Monoterpenes hydrocarbons } & $82.392 \pm 2.400 \mathrm{ab}$ & $93.234 \pm 2.716 \mathrm{a}$ & $58.633 \pm 1.708 \mathrm{~d}$ & $66.191 \pm 0.331 \mathrm{~cd}$ & $76.042 \pm 1.999 \mathrm{bc}$ & $88.82 \pm 2.587 \mathrm{a}$ & $60.808 \pm 1.771 \mathrm{~d}$ & $76.525 \pm 0.159 \mathrm{bc}$ \\
\hline \multicolumn{2}{|c|}{ Sesquiterpenes hydrocarbons } & $5.081 \pm 0.148 \mathrm{a}$ & $1.616 \pm 0.047 \mathrm{c}$ & $1.171 \pm 0.034 \mathrm{c}$ & $5.170 \pm 0.149 \mathrm{a}$ & $5.892 \pm 0.294 \mathrm{a}$ & $3.875 \pm 0.113 \mathrm{~b}$ & $4.957 \pm 0.144 \mathrm{a}$ & $5.298 \pm 0.072 \mathrm{a}$ \\
\hline
\end{tabular}


Table 6. Cont.

\begin{tabular}{|c|c|c|c|c|c|c|c|c|}
\hline \multirow[b]{2}{*}{ Compound } & \multicolumn{4}{|c|}{ Mountain } & \multicolumn{4}{|c|}{ Plain } \\
\hline & Summer & Autumn & Winter & Spring & Summer & Autumn & Winter & Spring \\
\hline Oxygenated monoterpenes & $1.640 \pm 0.048 \mathrm{bc}$ & $2.116 \pm 0.062 b$ & $1.912 \pm 0.056 \mathrm{~b}$ & $1.844 \pm 0.136 \mathrm{~b}$ & $1.521 \pm 0.234 \mathrm{bc}$ & $0.971 \pm 0.029 \mathrm{~cd}$ & $3.374 \pm 0.098 \mathrm{a}$ & $0.338 \pm 0.010 \mathrm{~d}$ \\
\hline Oxygenated sesquiterpenes & $12.273 \pm 0.358 \mathrm{e}$ & $5.302 \pm 0.154 \mathrm{f}$ & $39.772 \pm 1.159 \mathrm{a}$ & $24.718 \pm 0.544 \mathrm{c}$ & $13.409 \pm 0.986$ de & $7.832 \pm 0.228 \mathrm{f}$ & $29.793 \pm 0.868 b$ & $16.879 \pm 0.021 \mathrm{~d}$ \\
\hline Others & $1.352 \pm 0.040 \mathrm{a}$ & $0.663 \pm 0.020 \mathrm{bcd}$ & $0.484 \pm 0.014 \mathrm{~cd}$ & $1.081 \pm 0.045 \mathrm{abc}$ & $1.177 \pm 0.188 \mathrm{ab}$ & $1.340 \pm 0.039 \mathrm{a}$ & $1.502 \pm 0.044 \mathrm{a}$ & $0.405 \pm 0.042 \mathrm{~d}$ \\
\hline
\end{tabular}

Values $(n=3)$ in rows followed by the same letter are not significantly different, $p \leq 0.05$. In bold indicated EO components $>1 \%$.

Table 7. Chemical composition (\%) of essential oils of spearmint plants.

\begin{tabular}{|c|c|c|c|c|c|c|c|c|c|}
\hline \multirow[b]{2}{*}{ Compound } & \multirow[b]{2}{*}{ RI } & \multicolumn{4}{|c|}{ Mountain } & \multicolumn{4}{|c|}{ Plain } \\
\hline & & Summer & Autumn & Winter & Spring & Summer & Autumn & Winter & Spring \\
\hline$\alpha$ Pinene & 933 & $0.892 \pm 0.026 \mathrm{bc}$ & $0.835 \pm 0.025 c$ & $0.705 \pm 0.021 \mathrm{~d}$ & $1.003 \pm 0.022 \mathrm{ab}$ & $0.999 \pm 0.010 \mathrm{ab}$ & $1.039 \pm 0.030 \mathrm{a}$ & $0.918 \pm 0.027 \mathrm{ab}$ & $0.837 \pm 0.004 \mathrm{c}$ \\
\hline Camphene & 948 & $0.087 \pm 0.003 \mathrm{bc}$ & $0.095 \pm 0.003 \mathrm{bc}$ & $0.079 \pm 0.003 \mathrm{bc}$ & $0.158 \pm 0.017 \mathrm{a}$ & $0.068 \pm 0.001 \mathrm{c}$ & $0.082 \pm 0.003 \mathrm{bc}$ & $0.063 \pm 0.002 c$ & $0.104 \pm 0.001 \mathrm{~b}$ \\
\hline Sabinene & 973 & $0.586 \pm 0.017 \mathrm{~d}$ & $1.291 \pm 0.038 \mathrm{~d}$ & $15.547 \pm 0.453 a$ & $3.608 \pm 0.033 c$ & $0.687 \pm 0.018 d$ & $0.685 \pm 0.020 \mathrm{~d}$ & $4.798 \pm 0.140 \mathrm{~b}$ & $1.032 \pm 0.008 \mathrm{~d}$ \\
\hline$\beta$ Pinene & 977 & $1.200 \pm 0.035 \mathrm{~cd}$ & $1.238 \pm 0.036 \mathrm{bcd}$ & $1.165 \pm 0.034 \mathrm{~d}$ & $1.592 \pm 0.001 \mathrm{a}$ & $1.39 \pm 0.010 \mathrm{~b}$ & $1.358 \pm 0.040 \mathrm{bc}$ & $1.336 \pm 0.039 \mathrm{bc}$ & $1.299 \pm 0.004 \mathrm{bcd}$ \\
\hline$\beta$ Myrcene & 989 & $0.427 \pm 0.013 \mathrm{~d}$ & $0.570 \pm 0.017 \mathrm{c}$ & $0.335 \pm 0.010 \mathrm{de}$ & $0.739 \pm 0.031 \mathrm{ab}$ & $0.768 \pm 0.045 \mathrm{a}$ & $0.652 \pm 0.019 \mathrm{abc}$ & $0.269 \pm 0.008 \mathrm{e}$ & $0.634 \pm 0.004 \mathrm{bc}$ \\
\hline$\alpha$ Terpinene & 1005 & $0.062 \pm 0.002 c$ & $0.047 \pm 0.001 \mathrm{~cd}$ & $0.231 \pm 0.007 \mathrm{a}$ & $0.129 \pm 0.010 \mathrm{~b}$ & $0.043 \pm 0.005 \mathrm{~cd}$ & $0.022 \pm 0.001 \mathrm{~cd}$ & $0.134 \pm 0.004 \mathrm{~b}$ & $0.018 \pm 0.018 \mathrm{~d}$ \\
\hline D Limonene & 1028 & $5.185 \pm 0.151 b$ & $6.842 \pm 0.199 b$ & $4.091 \pm 0.119 \mathrm{~b}$ & $5.171 \pm 0.030 \mathrm{~b}$ & $14.603 \pm 1.313 \mathrm{a}$ & $11.913 \pm 0.347 \mathrm{a}$ & $4.731 \pm 0.138 b$ & $6.555 \pm 0.039 b$ \\
\hline 1,8-Cineole & 1031 & $5.034 \pm 0.147 \mathrm{~cd}$ & $6.157 \pm 0.180 \mathrm{~b}$ & $3.799 \pm 0.111 \mathrm{e}$ & $8.844 \pm 0.002 a$ & $6.516 \pm 0.417 b$ & $5.097 \pm 0.149 c$ & $3.995 \pm 0.117 \mathrm{de}$ & $8.206 \pm 0.039 a$ \\
\hline cis Ocimene & 1036 & $0.088 \pm 0.003$ def & $0.183 \pm 0.006 \mathrm{bc}$ & $0.076 \pm 0.002$ ef & $0.249 \pm 0.026 \mathrm{a}$ & $0.129 \pm 0.005$ cde & $0.133 \pm 0.004 \mathrm{~cd}$ & $0.073 \pm 0.002 \mathrm{f}$ & $0.228 \pm 0.002 \mathrm{ab}$ \\
\hline$\gamma$ Terpinene & 1058 & $0.124 \pm 0.004 c$ & $0.097 \pm 0.003 c$ & $0.486 \pm 0.014 \mathrm{a}$ & $0.268 \pm 0.017 \mathrm{~b}$ & $0.094 \pm 0.009 \mathrm{~cd}$ & $0.045 \pm 0.001 \mathrm{~d}$ & $0.281 \pm 0.008 b$ & $0.088 \pm 0.004 \mathrm{~cd}$ \\
\hline cis Sabinene hydrate & 1067 & $0.483 \pm 0.014 \mathrm{a}$ & $0.313 \pm 0.01 \mathrm{~b}$ & $0.098 \pm 0.003 \mathrm{~d}$ & $0.467 \pm 0.023 \mathrm{a}$ & $0.334 \pm 0.030 \mathrm{~b}$ & $0.199 \pm 0.006 c$ & $0 \pm 0 \mathrm{e}$ & $0.363 \pm 0.004 b$ \\
\hline $\begin{array}{c}\text { Isovaleric acid, } \\
\text { 2-methylbutyl ester }\end{array}$ & 1102 & $0.098 \pm 0.003 \mathrm{bc}$ & $0 \pm 0 \mathrm{c}$ & $0.122 \pm 0.004 \mathrm{~b}$ & $0.05 \pm 0.050 b c$ & $0 \pm 0 \mathrm{c}$ & $0 \pm 0 \mathrm{c}$ & $0.334 \pm 0.010 \mathrm{a}$ & $0.014 \pm 0.014 \mathrm{c}$ \\
\hline Borneol & 1166 & $0.220 \pm 0.006 \mathrm{abc}$ & $0.251 \pm 0.007 \mathrm{ab}$ & $0 \pm 0 \mathrm{c}$ & $0.212 \pm 0.119 \mathrm{abc}$ & $0.334 \pm 0.005 \mathrm{a}$ & $0.272 \pm 0.008 \mathrm{ab}$ & $0.082 \pm 0.003 \mathrm{bc}$ & $0.320 \pm 0.011 \mathrm{a}$ \\
\hline Terpinen-4-ol & 1178 & $0.297 \pm 0.009 \mathrm{bc}$ & $0.235 \pm 0.007$ bcde & $0.331 \pm 0.010 \mathrm{~b}$ & $0.539 \pm 0.070 \mathrm{a}$ & $0.295 \pm 0.007 \mathrm{bcd}$ & $0.120 \pm 0.004 \mathrm{e}$ & $0.155 \pm 0.005 \mathrm{de}$ & $0.175 \pm 0.004$ cde \\
\hline$\alpha$ Terpineol & 1191 & $0.061 \pm 0.002 c$ & $0.072 \pm 0.002 \mathrm{c}$ & $0 \pm 0 \mathrm{~d}$ & $0.016 \pm 0.016 \mathrm{~d}$ & $0.251 \pm 0.005 \mathrm{a}$ & $0.193 \pm 0.006 \mathrm{~b}$ & $0 \pm 0 \mathrm{~d}$ & $0.080 \pm 0.001 \mathrm{c}$ \\
\hline Dihydro carveol & 1194 & $16.250 \pm 0.474 \mathrm{a}$ & $13.272 \pm 0.387 b$ & $5.493 \pm 0.160 \mathrm{c}$ & $0.265 \pm 0.265 \mathrm{~d}$ & $0.292 \pm 0.076 \mathrm{~d}$ & $0.651 \pm 0.019 \mathrm{~d}$ & $6.887 \pm 0.201 c$ & $0.101 \pm 0.008 \mathrm{~d}$ \\
\hline
\end{tabular}


Table 7. Cont.

\begin{tabular}{|c|c|c|c|c|c|c|c|c|c|}
\hline \multirow[b]{2}{*}{ Compound } & \multirow[b]{2}{*}{ RI } & \multicolumn{4}{|c|}{ Mountain } & \multicolumn{4}{|c|}{ Plain } \\
\hline & & Summer & Autumn & Winter & Spring & Summer & Autumn & Winter & Spring \\
\hline neo Dihydro carveol & 1195 & $1.882 \pm 0.055 \mathrm{a}$ & $1.300 \pm 0.038 b$ & $0.789 \pm 0.023 \mathrm{~cd}$ & $0.450 \pm 0.214 \mathrm{de}$ & $0.409 \pm 0.034 \mathrm{de}$ & $0.391 \pm 0.011 \mathrm{de}$ & $1.104 \pm 0.033 b c$ & $0.197 \pm 0.004 \mathrm{e}$ \\
\hline trans Carveol & 1220 & $0.091 \pm 0.003 \mathrm{c}$ & $0.294 \pm 0.009 \mathrm{a}$ & $0 \pm 0 \mathrm{~d}$ & $0 \pm 0 \mathrm{~d}$ & $0.236 \pm 0.013 b$ & $0 \pm 0 \mathrm{~d}$ & $0.063 \pm 0.002 c$ & $0 \pm 0 \mathrm{~d}$ \\
\hline cis Carveol & 1231 & $0.697 \pm 0.021 \mathrm{c}$ & $3.674 \pm 0.107 \mathrm{a}$ & $2.546 \pm 0.074 b$ & $0.853 \pm 0.175 c$ & $0.384 \pm 0.075 \mathrm{~cd}$ & $0.131 \pm 0.004 \mathrm{~d}$ & $2.521 \pm 0.074 b$ & $0.183 \pm 0.028 \mathrm{~d}$ \\
\hline Pulegone & 1240 & $0.483 \pm 0.014 \mathrm{~b}$ & $0.448 \pm 0.013 b$ & $0.063 \pm 0.002 c$ & $0.085 \pm 0.027 c$ & $0.510 \pm 0.040 \mathrm{~b}$ & $0.724 \pm 0.021 \mathrm{a}$ & $0.127 \pm 0.004 c$ & $0.111 \pm 0.002 c$ \\
\hline Carvone & 1244 & $49.503 \pm 1.442 \mathrm{c}$ & $51.190 \pm 1.491 \mathrm{c}$ & $34.07 \pm 0.992 \mathrm{~d}$ & $64.525 \pm 0.688 b$ & $67.626 \pm 0.912 b$ & $74.794 \pm 2.179 \mathrm{a}$ & $44.937 \pm 1.309 \mathrm{c}$ & $67.042 \pm 0.139 \mathrm{~b}$ \\
\hline Isobornyl acetate & 1285 & $0.077 \pm 0.003 c$ & $0.087 \pm 0.003 \mathrm{c}$ & $0.367 \pm 0.011 b$ & $0 \pm 0 \mathrm{~d}$ & $0 \pm 0 \mathrm{~d}$ & $0 \pm 0 \mathrm{~d}$ & $0.453 \pm 0.013 \mathrm{a}$ & $0 \pm 0 \mathrm{~d}$ \\
\hline $\begin{array}{c}\text { iso Dihydro carveol } \\
\text { acetate }\end{array}$ & 1325 & $12.913 \pm 0.376 \mathrm{a}$ & $6.076 \pm 0.177 \mathrm{~d}$ & $10.846 \pm 0.316 b$ & $0.547 \pm 0.05 \mathrm{e}$ & $0.028 \pm 0.028 \mathrm{e}$ & $0.441 \pm 0.013 \mathrm{e}$ & $8.088 \pm 0.236 c$ & $0.103 \pm 0.004 \mathrm{e}$ \\
\hline trans Carvyl acetate & 1335 & $0 \pm 0 \mathrm{~d}$ & $0.247 \pm 0.008 c$ & $0.613 \pm 0.018 \mathrm{a}$ & $0 \pm 0 \mathrm{~d}$ & $0 \pm 0 \mathrm{~d}$ & $0 \pm 0 \mathrm{~d}$ & $0.495 \pm 0.015 b$ & $0 \pm 0 \mathrm{~d}$ \\
\hline cis Carvyl acetate & 1360 & $2.665 \pm 0.078 \mathrm{~d}$ & $4.982 \pm 0.146 c$ & $14.56 \pm 0.424 \mathrm{a}$ & $1.685 \pm 0.109 \mathrm{~d}$ & $0.06 \pm 0.022 \mathrm{e}$ & $0.279 \pm 0.008 \mathrm{e}$ & $12.514 \pm 0.365 b$ & $0.340 \pm 0.006 \mathrm{e}$ \\
\hline$\beta$ Bourbonene & 1386 & $0.634 \pm 0.019 \mathrm{~d}$ & $0.614 \pm 0.018 \mathrm{~d}$ & $0.913 \pm 0.027 c$ & $1.114 \pm 0.059 b$ & $0.684 \pm 0.004 \mathrm{~d}$ & $0.617 \pm 0.018 \mathrm{~d}$ & $1.403 \pm 0.041 \mathrm{a}$ & $1.534 \pm 0.007 \mathrm{a}$ \\
\hline$\beta$ Elemene & 1393 & $0.219 \pm 0.007 \mathrm{~d}$ & $0.162 \pm 0.005 \mathrm{~d}$ & $0.248 \pm 0.007 \mathrm{~cd}$ & $0.399 \pm 0.044 b$ & $0.216 \pm 0.018 \mathrm{~d}$ & $0.219 \pm 0.007 \mathrm{~d}$ & $0.340 \pm 0.010 \mathrm{bc}$ & $0.596 \pm 0.018 \mathrm{a}$ \\
\hline$\beta$ Caryophyllene & 1425 & $0.863 \pm 0.025 \mathrm{~d}$ & $0.876 \pm 0.026 \mathrm{~d}$ & $1.665 \pm 0.049 c$ & $2.648 \pm 0.088 b$ & $1.094 \pm 0.034 \mathrm{~d}$ & $0.911 \pm 0.027 \mathrm{~d}$ & $1.781 \pm 0.052 \mathrm{c}$ & $3.485 \pm 0.024 \mathrm{a}$ \\
\hline cis Cadina-1(6),4-diene & 1476 & $0.456 \pm 0.014 \mathrm{c}$ & $0.405 \pm 0.012 c$ & $0.731 \pm 0.022 \mathrm{~b}$ & $0.703 \pm 0.059 \mathrm{~b}$ & $0.384 \pm 0.021 \mathrm{c}$ & $0.434 \pm 0.013 \mathrm{c}$ & $0.793 \pm 0.023 b$ & $0.968 \pm 0.011 \mathrm{a}$ \\
\hline Germacrene D & 1497 & $0.454 \pm 0.014 \mathrm{c}$ & $0.287 \pm 0.009 \mathrm{~cd}$ & $0.153 \pm 0.005 \mathrm{de}$ & $1.512 \pm 0.082 b$ & $0.476 \pm 0.053 c$ & $0.394 \pm 0.012 c$ & $0 \pm 0 \mathrm{e}$ & $2.676 \pm 0.031 \mathrm{a}$ \\
\hline Bicyclogermacrene & 1512 & $0.177 \pm 0.005 \mathrm{c}$ & $0.129 \pm 0.004 \mathrm{~cd}$ & $0 \pm 0 \mathrm{~d}$ & $0.884 \pm 0.057 \mathrm{~b}$ & $0.237 \pm 0.023$ & $0.160 \pm 0.005 c$ & $0 \pm 0 \mathrm{~d}$ & $1.398 \pm 0.025 \mathrm{a}$ \\
\hline Germacrene A & 1519 & $0.051 \pm 0.002 \mathrm{bc}$ & $0.055 \pm 0.002 \mathrm{bc}$ & $0 \pm 0 \mathrm{c}$ & $0.078 \pm 0.038 \mathrm{~b}$ & $0.072 \pm 0.01 \mathrm{bc}$ & $0.075 \pm 0.002 \mathrm{bc}$ & $0.123 \pm 0.004 \mathrm{ab}$ & $0.165 \pm 0.002 \mathrm{a}$ \\
\hline trans Calamene & 1534 & $0.273 \pm 0.008 \mathrm{~d}$ & $0.195 \pm 0.006 \mathrm{e}$ & $0.478 \pm 0.014 \mathrm{~b}$ & $0.304 \pm 0.012 \mathrm{~d}$ & $0.205 \pm 0.009 \mathrm{e}$ & $0.244 \pm 0.007 \mathrm{de}$ & $0.588 \pm 0.017 \mathrm{a}$ & $0.375 \pm 0.010 \mathrm{c}$ \\
\hline Cubenol-1,10-di-epi & 1617 & $0.097 \pm 0.003 \mathrm{bc}$ & $0 \pm 0 \mathrm{c}$ & $0.152 \pm 0.004 \mathrm{~b}$ & $0.198 \pm 0.053 \mathrm{ab}$ & $0.104 \pm 0.008 \mathrm{bc}$ & $0.099 \pm 0.003 \mathrm{bc}$ & $0.293 \pm 0.009 \mathrm{a}$ & $0.172 \pm 0.012 \mathrm{~b}$ \\
\hline$a$ Cadinol & 1657 & $0 \pm 0 \mathrm{~b}$ & $0 \pm 0 \mathrm{~b}$ & $0 \pm 0 \mathrm{~b}$ & $0.088 \pm 0.031 \mathrm{a}$ & $0.100 \pm 0.006 \mathrm{a}$ & $0 \pm 0 \mathrm{~b}$ & $0 \pm 0 \mathrm{~b}$ & $0.076 \pm 0.005 \mathrm{a}$ \\
\hline \multicolumn{2}{|l|}{ Total Identified } & $99.893 \pm 0.020$ & $99.766 \pm 0.002$ & $98.165 \pm 0.020$ & $99.784 \pm 0.055$ & $99.896 \pm 0.003$ & $99.805 \pm 0.020$ & $97.4 \pm 0.020$ & $99.944 \pm 0.017$ \\
\hline \multicolumn{2}{|c|}{ Monoterpenes hydrocarbons } & $8.649 \pm 0.252 \mathrm{e}$ & $11.261 \pm 0.328$ de & $22.715 \pm 0.662 \mathrm{a}$ & $12.915 \pm 0.185 \mathrm{~cd}$ & $18.822 \pm 1.344 \mathrm{~b}$ & $15.961 \pm 0.465 \mathrm{bc}$ & $12.602 \pm 0.367 \mathrm{~d}$ & $10.793 \pm 0.066 \mathrm{de}$ \\
\hline \multicolumn{2}{|c|}{ Sesquiterpenes hydrocarbons } & $2.473 \pm 0.003 \mathrm{de}$ & $2.096 \pm 0.003 \mathrm{e}$ & $3.181 \pm 0.004 \mathrm{~cd}$ & $6.673 \pm 0.380 \mathrm{~b}$ & $2.682 \pm 0.166$ cde & $2.41 \pm 0.003 \mathrm{de}$ & $3.523 \pm 0.004 \mathrm{c}$ & $9.953 \pm 0.143 \mathrm{a}$ \\
\hline \multicolumn{2}{|c|}{ Oxygenated monoterpenes } & $73.197 \pm 0.073 \mathrm{c}$ & $75.124 \pm 0.075 \mathrm{bc}$ & $46.179 \pm 0.047 \mathrm{e}$ & $76.303 \pm 0.976 \mathrm{bc}$ & $77.28 \pm 1.477 \mathrm{ab}$ & $80.391 \pm 0.081 \mathrm{a}$ & $58.836 \pm 0.059 \mathrm{~d}$ & $76.789 \pm 0.113 \mathrm{~b}$ \\
\hline \multicolumn{2}{|c|}{ Oxygenated sesquiterpenes } & $0.094 \pm 0.000 \mathrm{bc}$ & $0 \pm 0 \mathrm{c}$ & $0.148 \pm 0.000 \mathrm{abc}$ & $0.285 \pm 0.083 \mathrm{a}$ & $0.203 \pm 0.013 \mathrm{ab}$ & $0.096 \pm 0.000 \mathrm{bc}$ & $0.284 \pm 0.000 \mathrm{a}$ & $0.248 \pm 0.018 \mathrm{ab}$ \\
\hline \multicolumn{2}{|l|}{ Others } & $15.317 \pm 0.016 c$ & $11.34 \pm 0.012 \mathrm{~d}$ & $25.716 \pm 0.026 \mathrm{a}$ & $2.402 \pm 0.166 \mathrm{e}$ & $0.226 \pm 0.045 \mathrm{~g}$ & $0.854 \pm 0.001 \mathrm{f}$ & $21.05 \pm 0.021 \mathrm{~b}$ & $0.508 \pm 0.014 \mathrm{fg}$ \\
\hline
\end{tabular}


3.4. Correlation of Antioxidant and Reducing Activity with Polyphenols, Minerals and Essential Oils Components

MAP species are valued as a natural source of antioxidants, with phenolic compounds and EO constituents playing an important role in this capacity $[19,27]$. Linear correlation coefficients were calculated and reported in detail in Tables S2-S4, to analyze the contribution of phenols, mineral content and EOs yield and components (only the three most prevalent elements of each EO were evaluated) to their total antioxidant capacity. The correlation coefficient $(r)$ and $p$-values between the examined EO compounds, the mineral content and the antioxidant capacity of sage are given in Table S2. In mountainous areas, in summer ABTS was negatively correlated with $\mathrm{N}$ content. EO yield was positively correlated with $\mathrm{Zn}$ but negatively with $\mathrm{Ca}, \mathrm{Mg}$ and Fe. In autumn, $\mathrm{EO}$ yield was positively correlated with $\mathrm{Mg}$ and Fe but negatively with $\mathrm{P}, \mathrm{Na}, \mathrm{Ca}$ and $\mathrm{Zn}$. In winter, phenols were positively correlated with ABTS, EO yield was positively correlated with Fe but negatively with $\mathrm{K}, \mathrm{P}$, $\mathrm{Ca}, \mathrm{Zn}$ and $\mathrm{Cu}$. In spring, FRAP was positively correlated with $\mathrm{P}, \mathrm{EO}$ yield was positively correlated with N, Ca and Cu but negatively with Fe and Zn (Table S2). Essential oil yield of Thymus migricus was fairly strongly related to the concentrations of minerals as Ca and $\mathrm{K}$ in soil, along with a series of soil properties such as the percentage of organic matter and soil texture, altitude and temperature [73].

In plain areas for summer sage, phenols were negatively correlated with 1,8-Cineole and Camphor, while EO yield was positively correlated with $\mathrm{N}, \mathrm{Mg}$ and Fe but negatively with nutrients as $\mathrm{K}, \mathrm{Ca}, \mathrm{Zn}$ and $\mathrm{Cu}$. In autumn, phenols were positively correlated with ABTS, and the EO yield was positively correlated with $\mathrm{P}, \mathrm{Na}, \mathrm{Fe}, \mathrm{Zn}$ and $\mathrm{Cu}$ but negatively with $\mathrm{Ca}$. In winter, sage EO yield was positively correlated with $\mathrm{K}, \mathrm{Ca}$, Fe and $\mathrm{Zn}$ but negatively with $\mathrm{Na}, \mathrm{Mg}$ and $\mathrm{Cu}$. In spring, FRAP was positively correlated with ABTS, while both FRAP and ABTS were negatively correlated with $\alpha$ Thujone. Antioxidant activity was also negatively linked to 1,8-Cineole and $\alpha$ Thujone in laurel and sage plants growing in Cyprus' plains, as well as to Neral, Geranial and Caryophyllene oxide in lemon verbena plants grown Cyprus' mountains [34]. EO yield was positively correlated with Fe and $\mathrm{Cu}$ but negatively with $\mathrm{P}, \mathrm{Na}$ and $\mathrm{Ca}$ (Table S2). The low antioxidant activity of 1,8-Cineole [74] may justify the negative correlation with phenols values observed in our study.

The relevant correlation coefficient $(r)$ and $p$-values between the analyzed EO compounds, and the mineral content and the antioxidant capacity of sideritis are given in Table S3. In mountainous areas, in summer, the minerals ( $, \mathrm{K}, \mathrm{P}, \mathrm{Ca}, \mathrm{Mg}, \mathrm{Fe}, \mathrm{Zn}$ and $\mathrm{Cu}$ ) were positively correlated with the sideritis EOs major components, namely $\alpha$ Pinene, Valeranone and $\beta$ Phellandrene. Additionally, the EO yield was positively correlated with $\mathrm{N}, \mathrm{K}$ and $\mathrm{Ca}$. In autumn, $\mathrm{Ca}, \mathrm{Mg}$, and $\mathrm{Zn}$ and the $\mathrm{EO}$ yield were positively correlated but $\mathrm{N}, \mathrm{K}, \mathrm{P}, \mathrm{Fe}$ and $\mathrm{Cu}$ were negatively correlated with the EO components such as $\alpha$ Pinene, Valeranone and $\beta$ Phellandrene. Additionally, the sideritis EO yield was positively correlated with $\mathrm{Zn}$ but negatively with $\mathrm{N}, \mathrm{K}, \mathrm{P}, \mathrm{Na}$, Fe and $\mathrm{Cu}$. In winter, ABTS was positively correlated with $\mathrm{N}$, while $\mathrm{K}, \mathrm{P}$, and $\mathrm{Zn}$ and the $\mathrm{EO}$ yield were positively correlated but $\mathrm{Cu}$ was negatively correlated with $\alpha$ Pinene, Valeranone and $\beta$ Phellandrene. Moreover, the EO yield was positively correlated with $\mathrm{K}, \mathrm{P}$ and $\mathrm{Zn}$ but negatively with $\mathrm{Cu}$. In spring, FRAP was positively correlated with ABTS. K, Na, Ca and Zn were positively correlated with $\alpha$ Pinene and $\beta$ Phellandrene, and negatively with Valeranone. However, the opposite was noticed in the case of $\mathrm{Cu}$, which was positively correlated with Valeranone and negatively with $\alpha$ Pinene and $\beta$ Phellandrene. EO yield was positively correlated with $\mathrm{P}$ (Table S3). In thyme, the content of Linalool was positively correlated with the percentages of silt and clay, and negatively with the concentrations of $\mathrm{Ca}, \mathrm{K}$ and $\mathrm{P}$, the percentages of sand and organic matter, and temperature. The opposite trend was evidenced for Thymol content [73], indicating the interaction of the EOs composition with nutrients availability of the growing substrate and the environmental conditions of plant growth.

In plain areas for sideritis, in summer, phenols were positively correlated with FRAP and ABTS, while positive correlation was found between FRAP and ABTS. K, P, Mg and Zn 
were negatively correlated $\alpha$ Pinene, and $\beta$ Phellandrene and positively with Valeranone. However, the opposite was found in the case of $\mathrm{Ca}, \mathrm{Cu}$ and $\mathrm{EO}$ yield, which were positively correlated with $\alpha$ Pinene and $\beta$ Phellandrene. EO yield was positively correlated with Ca and $\mathrm{Cu}$, but negatively with $\mathrm{K}, \mathrm{P}$ and $\mathrm{Mg}$. In autumn, phenols were positively correlated with FRAP. $\mathrm{Ca}, \mathrm{Mg}, \mathrm{Fe}, \mathrm{Zn}$, and $\mathrm{Cu}$ and $\mathrm{EO}$ yield were positively correlated but $\mathrm{K}, \mathrm{P}$, and $\mathrm{Na}$ were negatively correlated with $\alpha$ Pinene, Valeranone and $\beta$ Phellandrene. Similar to the individual $\mathrm{EO}$ components, $\mathrm{EO}$ yield was also positively correlated with $\mathrm{Ca}, \mathrm{Mg}, \mathrm{Fe}$, $\mathrm{Zn}$ and $\mathrm{Cu}$ and negatively correlated with $\mathrm{K}, \mathrm{P}$ and $\mathrm{Na}$. In winter, FRAP was positively correlated with ABTS. P and Ca were positively but $\mathrm{K}, \mathrm{Fe}, \mathrm{Zn}$ and $\mathrm{Cu}$ were negatively correlated with $\alpha$ Pinene, Valeranone and $\beta$ Phellandrene. In spring, FRAP was positively correlated with ABTS. P and $\mathrm{Mg}$ as well as EO yield were positively but $\mathrm{Ca}$ and $\mathrm{Fe}$ were negatively correlated with $\alpha$ Pinene, Valeranone and $\beta$ Phellandrene. EO yield was positively correlated with $\mathrm{P}$ and $\mathrm{Mg}$ and negatively with $\mathrm{Ca}$ and Fe (Table S3).

In spearmint, in mountainous areas in summer, $\mathrm{K}$ and $\mathrm{Na}$ content in plants were positively correlated with Carvone, Dihydro carveol and Sabinene, $\mathrm{N}$ was positively correlated with Sabinene, $\mathrm{Zn}$ and $\mathrm{Cu}$ were positively correlated with Carvone and Dihydro carveol, while EO yield was positively correlated with Dihydro carveol levels. EO yield were positively correlated $\mathrm{K}, \mathrm{Zn}$ and $\mathrm{Cu}$ but negatively correlated with $\mathrm{P}, \mathrm{Ca}$ and $\mathrm{Mg}$. In autumn, phenols were positively correlated with ABTS and Na. Moreover, $\mathrm{K}$ and $\mathrm{Zn}$ were positively correlated with Carvone, Dihydro carveol and Sabinene, while $\mathrm{Cu}$ was negatively correlated with Sabinene. Considering EO yield, Zn was positively but N, P, $\mathrm{Mg}$, Fe and $\mathrm{Cu}$ were negatively correlated with spearmint $\mathrm{EO}$ yield. In winter, Carvone, Dihydro carveol and Sabinene were positively correlated with the levels of N, P, Mg and Fe. In spring, Dihydro carveol was positively correlated with $\mathrm{K}, \mathrm{P}, \mathrm{Fe}, \mathrm{Zn}$ and $\mathrm{Cu}$ but negatively with Ca (Table S3).

Spearmint grown in plain area, in summer revealed a negative correlation between ABTS and N levels. Moreover, N, K and Fe were positively correlated with Sabinene and negative correlated with Dihydro carveol, while $\mathrm{Ca}, \mathrm{Cu}$ and $\mathrm{EO}$ yield were positive correlated with Dihydro carveol. $\mathrm{Mg}$ and $\mathrm{Cu}$ were positively but $\mathrm{P}$ and $\mathrm{Zn}$ were negatively correlated with EO yield. In summer, phenols were positive correlated with EO yield but negatively correlated with K, P and Zn. FRAP was positively correlated with Fe. $\mathrm{Ca}, \mathrm{Mg}$ and $\mathrm{Cu}$ were positively corelated with Carvone, Dihydro carveol and Sabinene, while Dihydro carveol was negatively correlated with Zn but positively with the EO yield. Moreover, EO yield was positively correlated with $\mathrm{Ca}, \mathrm{Mg}$ and $\mathrm{Cu}$ but negatively with $\mathrm{Zn}$. No correlation among phenols, antioxidants, EO yield and components could be found during winter in plain area for spearmint. In spring, $\mathrm{Na}, \mathrm{Ca}, \mathrm{Mg}$ and Fe were positively correlated but $\mathrm{Cu}$ and EO yield were negatively correlated with Dihydro carveol. EO yield was positively correlated with $\mathrm{Na}$ and $\mathrm{Cu}$ and negatively correlated with $\mathrm{Ca}, \mathrm{Mg}$ and Fe (Table S3).

Depending on the species and their growing environment, EOs may contribute to the total antioxidant activity. Figure 1 summarizes the most relevant correlations between antioxidant activity and chemical composition, as well as EO yield and component content. Total phenols and antioxidant activity (FRAP and ABTS) were stimulated in winter and spring in sage and spearmint, respectively, in mountainous areas (Figure 1). In sideritis, total phenols and antioxidants were stimulated mainly in winter in plain areas.

Increased EO yield in sage was observed in the summer-autumn period in plain areas, which was positively correlated with the increased levels of Camphor (Figure 1). For sage plants grown in plain areas in spring, FRAP and ABTS were negatively correlated with $\alpha$ Thujone, being in agreement with previous reports on the negative correlation of $\alpha$ Thujone and antioxidants (assayed DPPH) [34]; however, $\alpha$ Thujone alone is reported to possess antibacterial, cytotoxic and antiviral activities [75]. Higher temperatures may also favor the activity of the enzymes responsible for the synthesis of the terpenes, the main compounds of EOs [76]. It is widely known that high levels of Camphor are toxic [77] while EO with high proportions of Camphor are used in the phytosanitary industry [78]. Sideritis 
increased EO yield in autumn in the mountainous areas. According to Salehi et al. [74], Pinenes have considerable bioactive properties and their increased content in mountainousspring and plain-winter cultivated sideritis plants could partially justify the increased antioxidant activity (See Table 3). In spearmint, increased EO yield was found in summer, in plain areas (Figure 1). Spearmint plants, when subjected to several stress factors such as salinity and $\mathrm{Cu}$ toxicity, revealed oxidative damage and had lower levels of antioxidants and 1,8-Cineole in their leaves than plants that were not stressed [79], while the levels of 1,8-Cineole were positively correlated to flavanols [34].
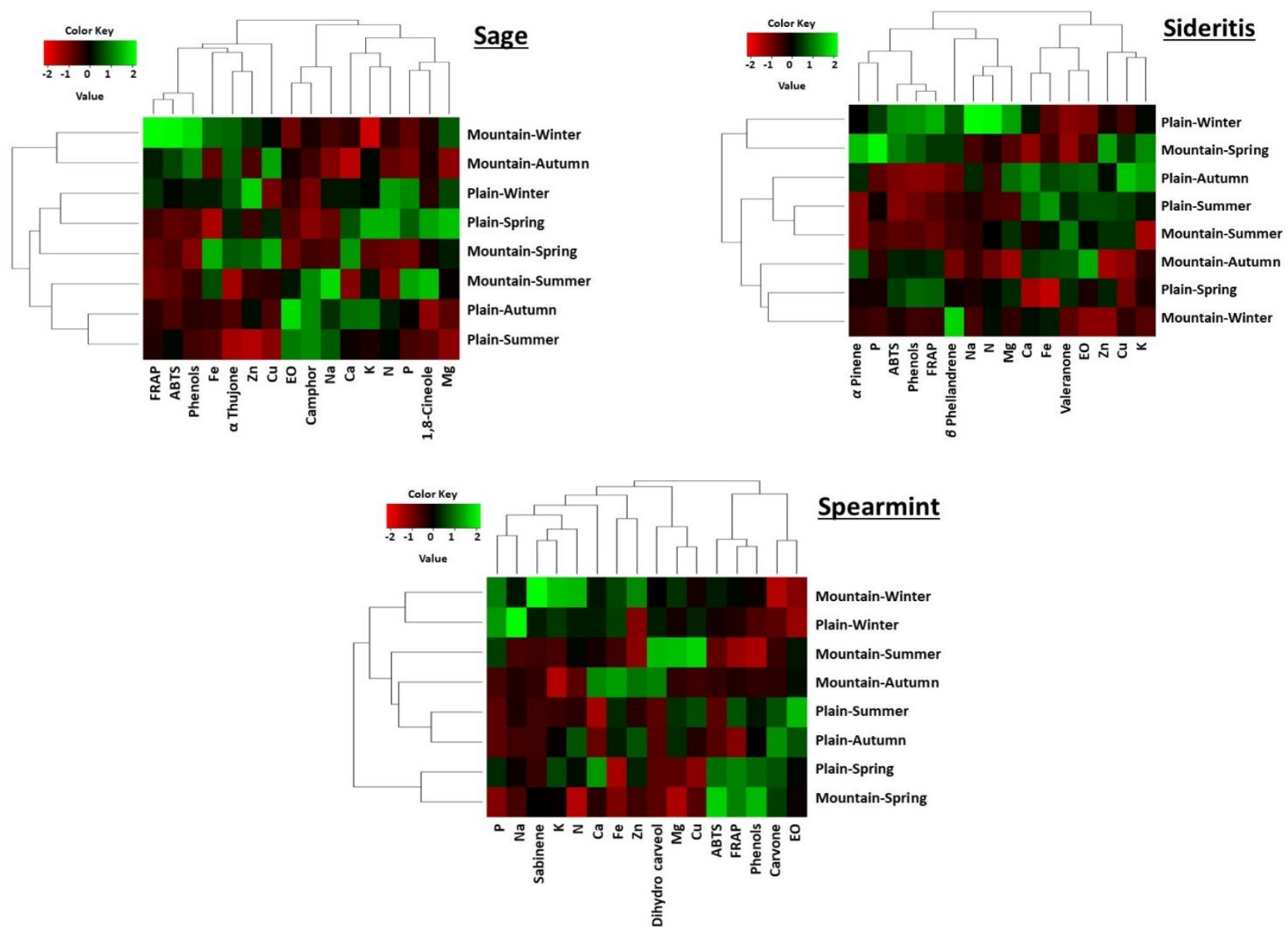

Figure 1. Relative changes (Heat maps) in total phenols (mg GAE $\left.\mathrm{g}^{-1} \mathrm{Fw}\right)$, antioxidant and reducing activity (FRAP, ABTS, mg trolox $\left.\mathrm{g}^{-1} \mathrm{Fw}\right)$, macronutrient $\left(\mathrm{g} \mathrm{kg}^{-1}\right)$ and micronutrient content $\left(\mathrm{mg} \mathrm{kg}^{-1}\right)$ content, and essential oil (EO) yield (\%) and three major EO components in sage, sideritis and spearmint as affected by the altitude (mountain vs. plain) and season (summer, autumn, winter, spring). Red shades indicate the lower level (less than -2.0 fold), deep red corresponds to -1.0 fold, black signifies that the level is not different from the mean value, deep green corresponds to 1.0 fold, clear green indicates that the level is more than 2.0 fold higher than the mean value.

These data suggest that, depending on the species and the environment where plants grow, EOs may add to total antioxidant activity. However, it should be noted that in our study, correlation analysis was performed only using data from the most abundant components of each species' EOs, which reduces the effect of the compounds with lower participation in the essential oil profile, in terms of antioxidant activity. It is well recognized that synergistic effects can exist among the components of natural matrices, and minor components may become critical for plant tissue antioxidant capability [80,81]. As a result, EOs may have greater antioxidant activity than isolated components, as Wang et al. [82] discovered for rosemary EO. Conjugated double bonds and phenolic groups, both of which have functional and antioxidant properties, may be found in essential oils [80]. 


\section{Conclusions}

In the present study, the levels of total phenols, antioxidant activity and mineral content of sage, sideritis and spearmint were investigated in terms of the impact of environmental condition-altitude (mountainous versus plain areas) and season (summer, autumn, winter and spring) as well as their correlation to the EO yield and composition. Season affected the total phenolic compounds content and antioxidant capacity, revealing increased values during winter and lower values during summer. Moreover, in winter, $\mathrm{P}$ and $\mathrm{Na}$ were the most accumulated minerals in the plant tissue but in summer, Fe was found to be accumulated more and the EO yield was increased. Altitude did not affect total phenolics and antioxidant compounds in a great extent, throughout a year period, but it did increase Fe content in mountainous plant species, while high levels of N, K, $\mathrm{Na}$ and $\mathrm{Ca}$ were obtained from plants grown in the plain areas. EO yield was varied in the different altitudes; EO composition was significantly affected as well. The highest antioxidant capacity, Zn content and EO yield were observed in sage, increased Fe content was found in sideritis, while spearmint plants revealed high N, Na and Mg levels. The EO yield and composition were correlated with various minerals, depending on the species. Plant antioxidant activity was positively linked to the total phenolic content, and in some cases with particular minerals (i.e., $\mathrm{P}$ in sage, $\mathrm{N}$ in sideritis), but negatively in some other cases (i.e., $\mathrm{N}$ in sage and spearmint) or constituents of the $\mathrm{EO}$ of the species (i.e., $\alpha$ Thujone in sage), although a varied response was observed depending on the species, season and altitude. In conclusion, the effect of different climatic conditions-altitudes and season on the antioxidant capacity, mineral content, EO yield and oils' composition of the researched MAPs in a species-dependent way may considerably alter plants secondary metabolites composition. These findings can be utilized to pinpoint certain sites and ecosystems but also to provide the appropriate fertilization on the cultivated species, where selected MAP cultures could be used to produce high-value crops with better quality and higher bioactive properties.

Supplementary Materials: The following are available online at https:/ /www.mdpi.com/article/10 .3390 /agronomy11091766/s1, Table S1: Fertilizers and crop protection means applied during the 1 year cultivation period. Table S2: Correlations coefficients and ( $p$-values) between the antioxidant activity and essential oils components of sage. Table S3: Correlations coefficients and ( $p$-values) between the antioxidant activity and essential oils components of Sideritis. Table S4: Correlations coefficients and ( $p$-values) between the antioxidant activity and essential oils components of spearmint. Figure S1: Meteorological data of the last 40 years (1972-2012 for the mountain and plain areas of the study. Meteorological data were obtained by the Department of Meteorology of Cyprus.

Author Contributions: Conceptualization, A.C. and N.T.; methodology, A.C.; software, A.C.; validation, E.E., A.C. and N.T.; formal analysis, A.C.; investigation, A.C.; resources, E.E. and N.T.; data curation, A.C., E.E., N.T.; writing—original draft preparation, A.C., E.E. and N.T.; writing—review and editing, A.C. and N.T.; visualization, A.C.; supervision, A.C.; project administration, N.T.; funding acquisition, N.T. All authors have read and agreed to the published version of the manuscript.

Funding: This research has been co-financed by the project AgroLabs that has been developed under the Programme Interreg V-B Balkan-Mediterranean 2014-2020, co-funded by the European Union and National Funds of the participating countries. Cyprus University of Technology Open Access Author Fund.

Conflicts of Interest: The authors declare no conflict of interest.

\section{References}

1. Raut, J.S.; Karuppayil, S.M. A status review on the medicinal properties of essential oils. Ind. Crop. Prod. 2014, 62, 250-264. [CrossRef]

2. Li, Y.; Kong, D.; Fu, Y.; Sussman, M.R.; Wu, H. The effect of developmental and environmental factors on secondary metabolites in medicinal plants. Plant Physiol. Biochem. 2020, 148, 80-89. [CrossRef]

3. Fares, R.; Bazzi, S.; Baydoun, S.E.; Abdel-Massih, R.M. The Antioxidant and anti-proliferative activity of the Lebanese Olea europaea extract. Plant Foods Hum. Nutr. 2011, 66, 58-63. [CrossRef] [PubMed] 
4. Máthé, A. Medicinal and Aromatic Plants of the World: Scientific, Production, Commercial and Utilization Aspects, Medicinal and Aromatic Plants of the World; Springer: Dordrecht, The Netherlands, 2015.

5. Krishnaiah, D.; Sarbatly, R.; Bono, A. Phytochemical antioxidants for health and medicine-A move towards nature. Biotechnol. Mol. Biol. Rev. 2007, 2, 97-104.

6. Nićiforović, N.; Mihailović, V.; Mašković, P.; Solujić, S.; Stojković, A.; Muratspahić, D.P. Antioxidant activity of selected plant species; potential new sources of natural antioxidants. Food Chem. Toxicol. 2010, 48, 3125-3130. [CrossRef]

7. Petropoulos, S.A.; Fernandes, Â.; Tzortzakis, N.; Sokovic, M.; Ciric, A.; Barros, L.; Ferreira, I.C.F.R. Bioactive compounds content and antimicrobial activities of wild edible Asteraceae species of the Mediterranean flora under commercial cultivation conditions. Food Res. Int. 2019, 119, 859-868. [CrossRef] [PubMed]

8. Vanzani, P.; Rossetto, M.; De Marco, V.; Sacchetti, L.E.; Paoletti, M.G.; Rigo, A. Wild Mediterranean Plants as Traditional Food: A Valuable Source of Antioxidants. J. Food Sci. 2011, 76, C46-C51. [CrossRef] [PubMed]

9. Gouthamchandra, K.; Mahmood, R.; Manjunatha, H. Free radical scavenging, antioxidant enzymes and wound healing activities of leaves extracts from Clerodendrum infortunatum L. Environ. Toxicol. Pharmacol. 2010, 30, 11-18. [CrossRef]

10. Başkan, S.; Öztekin, N.; Erim, F.B. Determination of carnosic acid and rosmarinic acid in sage by capillary electrophoresis. Food Chem. 2007, 101, 1748-1752. [CrossRef]

11. Kofidis, G.; Bosabalidis, A.M. Effects of altitude and season on glandular hairs and leaf structural traits of Nepeta nuda L. Bot. Stud. 2008, 49, 363-372.

12. Grausgruber-Gröger, S.; Schmiderer, C.; Steinborn, R.; Novak, J. Seasonal influence on gene expression of monoterpene synthases in Salvia officinalis (Lamiaceae). J. Plant Physiol. 2012, 169, 353-359. [CrossRef]

13. Blank, A.F.; Costa, A.G.; Arrigoni-Blank, M.D.F.; Cavalcanti, S.C.H.; Alves, P.B.; Innecco, R.; Ehlert, P.A.D.; De Sousa, I.F. Influence of season, harvest time and drying on Java citronella (Cymbopogon winterianus Jowitt) volatile oil. Rev. Bras. Farmacogn. 2007, 17, 557-564. [CrossRef]

14. Gonçalves, S.; Gomes, D.; Costa, P.; Romano, A. The phenolic content and antioxidant activity of infusions from Mediterranean medicinal plants. Ind. Crop. Prod. 2013, 43, 465-471. [CrossRef]

15. Dinu, C.; Vasile, G.-G.; Buleandra, M.; Popa, D.E.; Gheorghe, S.; Ungureanu, E.-M. Translocation and accumulation of heavy metals in Ocimum basilicum L. plants grown in a mining-contaminated soil. J. Soils Sediments 2020, 20, 2141-2154. [CrossRef]

16. Lajayer, B.A.; Ghorbanpour, M.; Nikabadi, S. Heavy metals in contaminated environment: Destiny of secondary metabolite biosynthesis, oxidative status and phytoextraction in medicinal plants. Ecotoxicol. Environ. Saf. 2017, 145, 377-390. [CrossRef] [PubMed]

17. Edris, A.E. Pharmaceutical and therapeutic potentials of essential oils and their individual volatile constituents: A review. Phytother. Res. 2007, 21, 308-323. [CrossRef]

18. Chen, Y.F.; Roan, H.Y.; Lii, C.K.; Huang, Y.C.; Wang, T.S. Relationship between antioxidant and antiglycation ability of saponins, polyphenols, and polysaccharides in Chinese herbal medicines used to treat diabetes. J. Med. Plants Res. 2011, 5, $2322-2331$.

19. Chrysargyris, A.; Xylia, P.; Botsaris, G.; Tzortzakis, N. Antioxidant and antibacterial activities, mineral and essential oil composition of spearmint (Mentha spicata L.) affected by the potassium levels. Ind. Crop. Prod. 2017, 103, 202-212. [CrossRef]

20. Tzortzakis, N.; Chrysargyris, A.; Sivakumar, D.; Loulakakis, K. Vapour or dipping applications of methyl jasmonate, vinegar and sage oil for pepper fruit sanitation towards grey mould. Postharvest Biol. Technol. 2016, 118, 120-127. [CrossRef]

21. Xylia, P.; Chrysargyris, A.; Botsaris, G.; Tzortzakis, N. Potential application of spearmint and lavender essential oils for assuring endive quality and safety. Crop. Prot. 2017, 102, 94-103. [CrossRef]

22. Xylia, P.; Clark, A.; Chrysargyris, A.; Romanazzi, G.; Tzortzakis, N. Quality and safety attributes on shredded carrots by using Origanum majorana and ascorbic acid. Postharvest Biol. Technol. 2019, 155, 120-129. [CrossRef]

23. Pandey, A.K.; Kumar, P.; Singh, P.; Tripathi, N.N.; Bajpai, V.K. Essential oils: Sources of antimicrobials and food preservatives. Front. Microbiol. 2017, 7, 2161. [CrossRef] [PubMed]

24. Litskas, V.; Chrysargyris, A.; Stavrinides, M.; Tzortzakis, N. Water-energy-food nexus: A case study on medicinal and aromatic plants. J. Clean. Prod. 2019, 233, 1334-1343. [CrossRef]

25. Moradi, P.; Ford-Lloyd, B.; Pritchard, J. Plant-water responses of different medicinal plant thyme (Thymus spp.) species to drought stress condition. Aust. J. Crop. Sci. 2014, 8, 666-673.

26. Marshall, E. Health and Wealth from Medicinal Aromatic Plants; FAO: Rome, Italy, 2011; ISBN 9789251070703.

27. Chrysargyris, A.; Kloukina, C.; Vassiliou, R.; Tomou, E.-M.; Skaltsa, H.; Tzortzakis, N. Cultivation strategy to improve chemical profile and anti-oxidant activity of Sideritis perfoliata L. subsp. perfoliata. Ind. Crop. Prod. 2019, 140, 111694. [CrossRef]

28. Jash, S.; Gorai, D.; Roy, R. Salvia genus and triterpenoids. Int. J. Pharm. Sci. Res. 2016, 7, 4710-4732.

29. Mamadalieva, N.Z.; Hussain, H.; Xiao, J. Recent advances in genus Mentha: Phytochemistry, antimicrobial effects, and food applications. Food Front. 2020, 1, 435-458. [CrossRef]

30. González-Burgos, E.; Carretero, M.E.; Gómez-Serranillos, M.P. Sideritis spp.: Uses, chemical composition and pharmacological activities-A review. J. Ethnopharmacol. 2011, 135, 209-225. [CrossRef] [PubMed]

31. Ben Farhat, M.; Landoulsi, A.; Chaouch-Hamada, R.; Sotomayor, J.A.; Jordán, M.J. Profiling of essential oils and polyphenolics of Salvia argentea and evaluation of its by-products antioxidant activity. Ind. Crop. Prod. 2013, 47, 106-112. [CrossRef]

32. Norani, M.; Ebadi, M.-T.; Ayyari, M. Volatile constituents and antioxidant capacity of seven Tussilago farfara L. populations in Iran. Sci. Hortic. 2019, 257, 108635. [CrossRef] 
33. Žugić, A.; Dordević, S.; Arsić, I.; Marković, G.; Živković, J.; Jovanović, S.; Tadić, V. Antioxidant activity and phenolic compounds in 10 selected herbs from Vrujci Spa, Serbia. Ind. Crop. Prod. 2014, 52, 519-527. [CrossRef]

34. Chrysargyris, A.; Mikallou, M.; Petropoulos, S.; Tzortzakis, N. Profiling of essential oils components and polyphenols for their antioxidant activity of medicinal and aromatic plants grown in different environmental conditions. Agronomy 2020, 10, 727. [CrossRef]

35. Kee, L.A.; Shori, A.B.; Baba, A.S. Bioactivity and health effects of Mentha spicata. Integr. Food Nutr. Metab. 2017, 5, 1-2. [CrossRef]

36. Chrysargyris, A.; Petropoulos, S.A.; Fernandes, Â.; Barros, L.; Tzortzakis, N.; Ferreira, I.C.F.R. Effect of phosphorus application rate on Mentha spicata L. grown in deep flow technique (DFT). Food Chem. 2019, 276, 84-92. [CrossRef] [PubMed]

37. Lall, N.; Chrysargyris, A.; Lambrechts, I.; Fibrich, B.; Van Staden, A.B.; Twilley, D.; de Canha, M.N.; Oosthuizen, C.B.; Bodiba, D.; Tzortzakis, N. Siderits perfoliata (subsp. perfoliata) nutritive value and its potential medicinal properties. Antioxidants $2019,8,521$. [CrossRef]

38. Ghorbani, A.; Esmaeilizadeh, M. Pharmacological properties of Salvia officinalis and its components. J. Tradit. Complement. Med. 2017, 7, 433-440. [CrossRef]

39. Baricevic, D.; Bartol, T. The biological/pharmacological activity of the Salvia Genus. V. Pharmacology. In Sage: The Genus Salvia; Kintzios, S., Ed.; Harwood Academic Publishers: Abingdon, UK, 2000; pp. 143-184. ISBN 0-203-34348-4.

40. Tzortzakis, N.G.; Tzanakaki, K.; Economakis, C.D. Effect of origanum oil and vinegar on the maintenance of postharvest quality of tomato. Food Nutr. Sci. 2011, 2, 974-982. [CrossRef]

41. Chrysargyris, A.; Nikou, A.; Tzortzakis, N. Effectiveness of Aloe vera gel coating for maintaining tomato fruit quality. N. Z. J. Crop. Hortic. Sci. 2016, 44, 203-217. [CrossRef]

42. Wojdyło, A.; Oszmiański, J.; Czemerys, R. Antioxidant activity and phenolic compounds in 32 selected herbs. Food Chem. 2007, 105, 940-949. [CrossRef]

43. Chrysargyris, A.; Prasad, M.; Kavanagh, A.; Tzortzakis, N. Biochar type, ratio, and nutrient levels in growing media affects seedling production and plant performance. Agronomy 2020, 10, 1421. [CrossRef]

44. Chrysargyris, A.; Loupasaki, S.; Petropoulos, S.A.; Tzortzakis, N. Salinity and cation foliar application: Implications on essential oil yield and composition of hydroponically grown spearmint plants. Sci. Hortic. 2019, 256, 108581. [CrossRef]

45. Pandey, K.B.; Rizvi, S.I. Plant polyphenols as dietary antioxidants in human health and disease. Oxid. Med. Cell. Longev. 2009, 2, 270-278. [CrossRef]

46. Lemos, M.F.; Lemos, M.F.; Pacheco, H.P.; Endringer, D.C.; Scherer, R. Seasonality modifies rosemary's composition and biological activity. Ind. Crop. Prod. 2015, 70, 41-47. [CrossRef]

47. Majuakim, L.; Ng, S.Y.; Fadzelly, M.; Bakar, A.; Suleiman, M. Effect of altitude on total phenolics and flavonoids in Sphagnum junghuhnianum in tropical montane forests of Borneo. Sepilok Bull. 2014, 32, 23-32.

48. Pandey, G.; Khatoon, S.; Pandey, M.M.; Rawat, A.K.S. Altitudinal variation of berberine, total phenolics and flavonoid content in Thalictrum foliolosum and their correlation with antimicrobial and antioxidant activities. J. Ayurveda Integr. Med. 2018, 9, 169-176. [CrossRef] [PubMed]

49. Esmaeili, H.; Karami, A.; Hadian, J.; Saharkhiz, M.J.; Ebrahimi, S.N. Variation in the phytochemical contents and antioxidant activity of Glycyrrhiza glabra populations collected in Iran. Ind. Crop. Prod. 2019, 137, 248-259. [CrossRef]

50. Nurzyńska-Wierdak, R.; Rozek, E.; Borowski, B. Response of different basil cultivars to nitrogen and potassium fertilization: Total and mineral nitrogen content in herb. Acta Sci. Pol. Hortorum Cultus 2011, 10, 217-232.

51. Puttanna, K.; Rao, E.V.S.P.; Singh, R.; Ramesh, S. Influence of nitrogen and potassium fertilization on yield and quality of rosemary in relation to harvest number. Commun. Soil Sci. Plant Anal. 2010, 41, 190-198. [CrossRef]

52. Barra, A. Factors affecting chemical variability of essential oils: A review of recent developments. Nat. Prod. Commun. 2009, 4, 1147-1154. [CrossRef]

53. Cruz, E.M.D.O.; Pinto, J.A.O.; Fontes, S.S.; Arrigoni-Blank, M.D.F.; Bacci, L.; De Jesus, H.C.R.; Santos, D.D.A.; Alves, P.B.; Blank, A.F. Water deficit and seasonality study on essential oil constituents of Lippia gracilis schauer germplasm. Sci. World J. 2014, 2014, 314626. [CrossRef]

54. Santos-Gomes, P.C.; Fernandes-Ferreira, M. Organ- and season-dependent variation in the essential oil composition of Salvia officinalis L. cultivated at two different sites. J. Agric. Food Chem. 2001, 49, 2908-2916. [CrossRef]

55. Yang, L.; Wen, K.-S.; Ruan, X.; Zhao, Y.-X.; Wei, F.; Wang, Q. Response of plant secondary metabolites to environmental factors. Molecules 2018, 23, 762. [CrossRef]

56. Formisano, C.; Delfine, S.; Oliviero, F.; Tenore, G.C.; Rigano, D.; Senatore, F. Correlation among environmental factors, chemical composition and antioxidative properties of essential oil and extracts of chamomile (Matricaria chamomilla L.) collected in Molise (South-central Italy). Ind. Crop. Prod. 2015, 63, 256-263. [CrossRef]

57. Mahdavi, M.; Jouri, M.H.; Mahmoudi, J.; Rezazadeh, F.; Mahzooni-Kachapi, S.S. Investigating the altitude effect on the quantity and quality of the essential oil in Tanacetum polycephalum Sch.-Bip. polycephalum in the Baladeh region of Nour, Iran. Chin. J. Nat. Med. 2013, 11, 553-559. [CrossRef] [PubMed]

58. Mahdavi, M.; Jouri, M.H.; Mahzooni-Kachapi, S.S.; Sadeghihardoroodi, M. The Effects of Altitude on Productivity and Formative Components of Essential Oils of Artemisia absinthium L. (Iran). Bull. Environ. Pharmacol. Life Sci. 2014, 3, $218-224$. 
59. Mahomoodally, F.; Aumeeruddy-Elalfi, Z.; Venugopala, K.N.; Hosenally, M. Antiglycation, comparative antioxidant potential, phenolic content and yield variation of essential oils from 19 exotic and endemic medicinal plants. Saudi J. Biol. Sci. 2019, 26, 1779-1788. [CrossRef] [PubMed]

60. Sabbahi, M.; El-Hassouni, A.; Tahani, A.; El-Bachiri, A. Volatile variability and antioxidant activity of Rosmarinus officinalis essential oil as affected by elevation gradient and vegetal associations. Asian J. Chem. 2019, 31, 1279-1288. [CrossRef]

61. Holopainen, J.K.; Himanen, S.J.; Yuan, J.S.; Chen, F.; Stewart, C.N. Ecological functions of terpenoids in changing climates. In Natural Products; Ramawat, K.G., Mérillon, J.M., Eds.; Springer: Berlin/Heidelberg, Germany, 2013.

62. Caldas, G.F.R.; Oliveira, A.R.D.S.; Araújo, A.V.; Lafayette, S.S.L.; Albuquerque, G.S.; Silva-Neto, J.D.C.; Costa-Silva, J.H.; Ferreira, F.; Da Costa, J.G.M.; Wanderley, A.G. Gastroprotective mechanisms of the monoterpene 1,8-cineole (eucalyptol). PLoS ONE 2015, 10, e0134558. [CrossRef]

63. Bedini, S.; Guarino, S.; Echeverria, M.C.; Flamini, G.; Ascrizzi, R.; Loni, A.; Conti, B. Allium sativum, Rosmarinus officinalis, and Salvia officinalis essential oils: A spiced shield against blowflies. Insects 2020, 11, 143. [CrossRef]

64. Cvetkovikj, I.; Stefkov, G.; Karapandzova, M.; Kulevanova, S.; Satovic, Z. Essential Oils and Chemical Diversity of Southeast European Populations of Salvia officinalis L. Chem. Biodivers. 2015, 12, 1025-1039. [CrossRef]

65. Samani, M.R.; Pirbalouti, A.G.; Moattar, F.; Golparvar, A.R. L-Phenylalanine and bio-fertilizers interaction effects on growth, yield and chemical compositions and content of essential oil from the sage (Salvia officinalis L.) leaves. Ind. Crop. Prod. 2019, 137, 1-8. [CrossRef]

66. Usano-Alemany, J.; Herraiz-Peñalver, D.; Cuadrado, J.; Díaz, S.; Santa-Cruz, M.; Palá-Paúl, J. Seasonal variation of the essential oils of Salvia lavandulifolia: Antibacterial activity. J. Essent. Oil-Bear. Plants 2012, 15, 195-203. [CrossRef]

67. Taie, H.A.A.; Salama, Z.A.E.R.; Radwan, S. Potential Activity of Basil Plants as a Source of Antioxidants and Anticancer Agents as Affected by Organic and Bio-organic Fertilization. Not. Bot. Horti Agrobot. Cluj-Napoca 2010, 38, 119-127.

68. Botrel, P.P.; Pinto, J.E.B.P.; Ferraz, V.; Bertolucci, S.K.V.; Figueiredo, F.C. Teor e composição química do óleo essencial de Hyptis marrubioides Epl., Lamiaceae em função da sazonalidade. Acta Sci.-Agron. 2010, 32, 533-538. [CrossRef]

69. Sgarbossa, J.; Schmidt, D.; Schwerz, F.; Schwerz, L.; Prochnow, D.; Caron, B.O. Effect of season and irrigation on the chemical composition of Aloysia triphylla essential oil. Rev. Ceres 2019, 66, 85-93. [CrossRef]

70. Elmastaş, M.; Dermirtas, I.; Isildak, O.; Aboul-Enein, H.Y. Antioxidant activity of S-carvone isolated from spearmint (Mentha spicata L. Fam Lamiaceae). J. Liq. Chromatogr. Relat. Technol. 2006, 29, 1465-1475. [CrossRef]

71. Wu, Z.; Tan, B.; Liu, Y.; Dunn, J.; Guerola, P.M.; Tortajada, M.; Cao, Z.; Ji, P. Chemical Composition and Antioxidant Properties of Essential Oils from Peppermint, Native Spearmint and Scotch Spearmint. Molecules 2019, 24, 2825. [CrossRef]

72. Talebi, S.M.; Nohooji, M.G.; Yarmohammadi, M.; Khani, M.; Matsyura, A. Effect of altitude on essential oil composition and on glandular trichome density in three Nepeta species (N. sessilifolia, N. heliotropifolia and N. fisSA). Mediterr. Bot. 2019, 40, 81-93. [CrossRef]

73. Yavari, A.; Nazeri, V.; Sefidkon, F.; Hassani, M.E. Influence of Some Environmental Factors on the Essential Oil Variability of Thymus migricus. Nat. Prod. Commun. 2010, 5, 943-948. [CrossRef]

74. Salehi, B.; Upadhyay, S.; Orhan, I.E.; Jugran, A.K.; Jayaweera, S.L.D.; Dias, D.A.; Sharopov, F.; Taheri, Y.; Martins, N.; Baghalpour, N.; et al. Therapeutic potential of $\alpha$-and $\beta$-pinene: A miracle gift of nature. Biomolecules 2019, 9, 738. [CrossRef]

75. Sivropoulou, A.; Nikolaou, C.; Papanikolaou, E.; Kokkini, S.; Lanaras, T.; Arsenakis, M. Antimicrobial, cytotoxic, and antiviral activities of Salvia fructicosa essential oil. J. Agric. Food Chem. 1997, 45, 3197-3201. [CrossRef]

76. De Oliveira, B.M.S.; Blank, A.F.; Nizio, D.A.D.C.; Nogueira, P.C.D.L.; Arrigoni-Blank, M.D.F.; Bacci, L.; Melo, C.R.; Nascimento, L.F.D.A.; Sampaio, T.S. Chemical analyses of the essential oils from Varronia curassavica accessions in two seasons. J. Essent. Oil Res. 2020, 32, 494-511. [CrossRef]

77. Chen, L.; Su, J.; Li, L.; Li, B.; Li, W. A new source of natural D-borneol and its characteristic. J. Med. Plants Res. 2011, 5, 3440-3447.

78. Kaloustian, J.; Pauli, A.-M.; Pastor, J. Evolution of camphor and others components in the essential oils of two labiate species during the biological cycle. Analusis 2000, 28, 308-315. [CrossRef]

79. Chrysargyris, A.; Papakyriakou, E.; Petropoulos, S.A.; Tzortzakis, N. The combined and single effect of salinity and copper stress on growth and quality of Mentha spicata plants. J. Hazard. Mater. 2019, 368, 584-593. [CrossRef]

80. Crespo, Y.A.; Sánchez, L.R.B.; Quintana, Y.G.; Cabrera, A.S.T.; del Sol, A.B.; Mayancha, D.M.G. Evaluation of the synergistic effects of antioxidant activity on mixtures of the essential oil from Apium graveolens L., Thymus vulgaris L. and Coriandrum sativum L. using simplex-lattice design. Heliyon 2019, 5, e01942. [CrossRef]

81. Fandiño, I.; Fernandez-Turren, G.; Ferret, A.; Moya, D.; Castillejos, L.; Calsamiglia, S. Exploring additive, synergistic or antagonistic effects of natural plant extracts on in vitro beef feedlot-type rumen microbial fermentation conditions. Animals 2020, 10, 173. [CrossRef]

82. Wang, Y.; Zhang, L.-T.; Feng, Y.-X.; Zhang, D.; Guo, S.-S.; Pang, X.; Geng, Z.-F.; Xi, C.; Du, S.-S. Comparative evaluation of the chemical composition and bioactivities of essential oils from four spice plants (Lauraceae) against stored-product insects. Ind. Crop. Prod. 2019, 140, 111640. [CrossRef] 